\title{
A CONVERGENCE THEORY FOR SADDLE FUNCTIONS ${ }^{1}$
}

\author{
BY
}

HEDY ATTOUCH AND ROGER J.-B. WETS

\begin{abstract}
We develop a convergence theory called epi/hypo-convergence, for bivariate functions that essentially implies the convergence of their saddle points. We study the properties of this limiting process in particular. We characterize the limit functions associated to any collection of bivariate functions and obtain a compactness theorem for the space of saddle functions. Even when restricted to the univariate case, the results generalize those known for epi-convergence. In particular, we show that the analysis of the convergence process via Yosida approximates must not be restricted to the convex case.
\end{abstract}

1. Introduction. In this paper we develop a convergence theory of the variationaltype for bivariate functions. We call it epi/hypo-convergence. It extends the theory of epi-convergence (for univariate functions), originally developed by Wijsman, Mosco, De Giorgi and his many collaborators, to study optimization problems (calculus of variations, variational inequalities,...).

The bivariate case, by which we simply mean a collection of functions $\left\{F_{\nu}, \nu \in N\right\}$ defined on a product space $X \times Y$, presents technical and conceptual difficulties not encountered when dealing with epi-convergence. In fact, the theory developed here stops far short from a complete answer to the (variational) convergence of bivariate functions. One might be interested in the convergence of the minimax of the functions $F_{v}$, or the maximin of these same functions. In general, the roles played by these quantities is asymmetric, and the convergence (existence,...) of one does not provide us with much information about the convergence (existence,...) of the other. Although most of our statements apply to any class of bivariate functions, the theory is intrinsically designed to handle bivariate functions that admit saddle points. This is more a matter of intent, than a precondition that needs to be imposed at the outset. To remind the reader of this concern, we do not hesitate to refer to functions defined on $X \times Y$ as saddle functions, although there is no implied assumption that they actually admit a saddle point!

We are motivated by approximation and limit problems that arise in Mechanics, Optimization, Hamiltonian Theory,... that cannot be handled in the framework of epi-convergence: in particular, by some problems connected to variational inequalities defined by maximal monotone operators that are not necessarily symmetric-for

Received by the editors August 20, 1981 and, in' revised form, August 5, 1982. 1980 Mathematics Subject Classification. Primary 49D99, 58E30; Secondary 54A20.

${ }^{1}$ Supported in part by a grant of the National Science Foundation. $0002-9947 / 83 \$ 1.00+\$ .25$ per page 
example certain classes of homogenization problems in Mechanics-also by convergence questions involving the Hamiltonian and Lagrangian functions generated by optimization problems (with state dynamics), and so on.

In order to set the stage we start in $\$ 2$ with a review of the theory of epi-convergence. We then introduced the definition of epi/hypo-convergence when the domain of the saddle functions is metrizable and we show that epi/hypo-convergence essentially implies the convergence of the saddle points; this is made precise in $\S 3$. Next, we study the limit functions, with respect to the topology of epi/hypo-convergence, asssociated to a collection of saddle functions. We see that the epi/hypo-limit, when it exists, is not necessarily unique. Thus the topology of epi/hypo-convergence is not separated (Hausdorff). In many ways this is not surprising. Bivariate functions, when viewed as saddle functions, are unaffected-as far as their operational characteristics are concerned-by a number of (closure) operations. It is this phenomena that shows up when taking epi/hypo-limits.

$\$ 5$ introduces and studies the Yosida approximates of saddle functions (in the metrizable case). We exhibit the relationship between epi/hypo-convergence and the convergence of the Yosida approximates. The results are new even when restricted to the univariate case because we make no convexity assumptions. Finally, in $\S 6$, we derive a compactness theorem. It shows that the class of saddle functions is compact under epi/hypo-convergence. In view of the results of $\$ 3$, this implies that if we have a sequence of bivariate functions that admit saddle points (clustering up to some point), then every associated epi/hypo-limit function will also admits saddle points. The limit functions are thus of the same nature as the elements of the sequence.

The definition of epi/hypo-convergence was given its first airing in [2]. A related, but stronger, notion of convergence for saddle functions was given by Sontag [12], however his attention is limited to a much more restricted class of bivariate functions. The work closest to ours is that of E. Cavazzuti [4]. He too deals with a stronger notion of convergence than that introduced here. The relationship between his work and ours will be made explicit towards the end of $\$ 4$. In addition, C. Sbordone (Napoli) indicated in his abstract for the meeting "Optimisation: Theorie et Algorithmes" (Confolant, Mars 1981) that he has used the theory of epi-convergence ( $\Gamma$-convergence) to obtain the convergence of saddle points.

2. Epi-convergence. We start with a quick review of the basic results for the univariate case. The theory provides a convergence theory for optimization or variational problems involving just minimization (or maximization). This is well documented; consult for example [9, 5, 1, 12 and 14]. This material will serve as background to the study of the bivariate case. In particular it allows us to bring to the attention of the reader the situation when the functions take on the value $-\infty$, i.e. are not proper. This has usually been glossed over in earlier presentation because for univariate functions this extreme situation presents no real practical interest. In the bivariate case, saddle functions take naturally the values $+\infty$ and $-\infty$ and we shall have to deal with their epi-convergence.

In this presentation we have chosen to deal with epi-convergence; every definition and every result has its counterpart in the setting of hypo-convergence. For the 
record we give the basic formulas for hypographs at the end of this section. However one should be aware of the asymmetric roles played by $+\infty$ and $-\infty$; in particular, our choice conditions the addition rules for the extended reals, viz $(+\infty)+a=+\infty$ for all $a \in \bar{R}$ and $(-\infty)+a=-\infty$ for all $a \in R \cup\{-\infty\}$. Every collection of extended-real numbers always has upper and lower bounds in $\bar{R}$, and thus in that sense liminf and lim sup are always well defined.

Let $f: X \rightarrow \bar{R}$ be an extended-real valued function defined on $X$, and $\tau$ a topology on $X ; \mathfrak{T}_{\tau}(x)$ is the neighborhood system of $x$. The effective domain of $f$ is $\operatorname{dom} f=$ $\{x \mid f(x)<+\infty\}$; it is proper if $f>-\infty$ and $\operatorname{dom} f \neq \varnothing$. The function is $\tau$-lower semicontinuous $(\tau$-1.s.c. or simply l.s.c.) if its epigraph

$$
\text { epi } f=\{(x, \alpha) \in X \times R \mid \alpha \geqslant f(x)\}
$$

is a closed subset of $X \times R$. The lower closure of $f$ is the function cl $f: X \rightarrow \bar{R}$ such that

$$
\text { epi cl } f=\operatorname{clepi} f \text {, }
$$

where cl epi $f$ is the closure of epi $f$ with respect to the product topology of $\tau$ and the natural topology on $R$. The so-defined function is also given by

$$
\operatorname{cl} f(x)=\sup _{V \in \mathscr{R}_{\tau}(x)} \inf _{y \in V} f(y)
$$

as follows from the following string of equivalent statements:

$$
\begin{aligned}
& (x, \alpha) \in \operatorname{clepi} f, \\
& \text { for all } \left.\left(V \in \Re_{\tau}(x), \varepsilon>0\right), \quad V \times\right] \alpha-\varepsilon, \alpha+\varepsilon[\cap \text { epi } f \neq \varnothing, \\
& \text { for all } \left.\left(V \in \Re_{\tau}(x), \varepsilon>0\right), \quad V \times\right]-\infty, \alpha+\varepsilon[\cap \text { epi } f \neq \varnothing, \\
& \text { for all }\left(V \in \Re_{\tau}(x), \varepsilon>0\right), \quad \exists y \in V \text { such that } f(y)<\alpha+\varepsilon, \\
& \text { for all } V \in \Re_{\tau}(x), \quad \inf _{y \in V} f(y) \leqslant \alpha, \quad \sup _{V \in \Re_{\tau}(x)} \inf _{y \in V} f(y) \leqslant \alpha, \\
& \text { cl } f(x) \leqslant \alpha \text {, i.e. }(x, \alpha) \in \operatorname{epi~cl~} f .
\end{aligned}
$$

Thus a function $f$ is $\tau$-lower semicontinuous if and ony if $f=\operatorname{cl} f$.

The extended lower closure of $f$ is the function cl $f$ defined as follows,

$$
\text { cl } f=\left[\begin{array}{ll}
\operatorname{cl} f & \text { if } \operatorname{cl} f>-\infty \\
-\infty & \text { otherwise. }
\end{array}\right.
$$

With these definitions we get

2.2 Proposition. Given a topological space $(X, \tau)$ and a function $f: X \rightarrow \bar{R}$, we have inf $f=\inf \mathrm{cl} f=\inf \mathrm{cl} f$. Moreover, for any open subset $G$ of $X$, we also have $\inf _{y \in G} f(y)=\inf _{y \in G} \mathrm{cl} f(\bar{y})$.

Proof. In view of the definition of $\mathrm{cl} f$, and the fact that $X$ is open it clearly suffices to prove the last identity. In fact, since cl $f \leqslant f$, we only need to show that $\inf _{G} f \leqslant \inf _{G} \mathrm{cl} f$ when $\inf _{G}>-\infty$. For all $y \in G, G \in \mathfrak{\Re}_{\tau}(y)$ since $G$ is open, and thus from (2.1) we get that $\operatorname{cl} f(y) \geqslant \inf _{u \in G} f(u)$. Since this holds for every $y \in G$, it follows that $\inf _{y \in G} \operatorname{cl} f(y) \geqslant \inf _{y \in G} f(y)$.

This innocuous proposition has numerous implications in the analysis of the convergence of infima. To begin with it will not matter if we work with $f, \operatorname{cl} f$ or $\operatorname{cl} f$. 
Also, all functions whose closure takes on anywhere the value $-\infty$, have the same extended lower closure. This suggests that we might be led to consider a variational convergence theory in terms of equivalence classes of functions. We shall need to take this viewpoint when dealing with bivariate (saddle) functions, at least in what we shall call the epi/hypo-limit of a sequence.

We first consider epi-limits in the sequential case. By this we mean that $(X, \tau)$ is metrizable and that we are given a sequence of functions $\left\{f_{\nu}: X \rightarrow \bar{R}, \nu=1, \ldots\right\}$. (All that is really needed at this stage is that locally $X$ admits a countable base.) The sequence $\left\{f_{\nu}, \nu=1, \ldots\right\}$ epi-converges to $f$ at $x$ if

for every subsequence of functions $\left\{f_{\nu_{k}}, k=1, \ldots\right\}$ and any sequence $\left\{x_{k}, k=1, \ldots\right\}$ converging to $x, \liminf _{k \rightarrow \infty} f_{\nu_{k}}\left(x_{k}\right) \geqslant$ $f(x)$, and there exists a sequence $\left\{x_{\nu}, \nu=1, \ldots\right\}$ converging to $x$ such that $\limsup f_{\nu}\left(x_{\nu}\right) \leqslant f(x)$.

$$
k \rightarrow \infty
$$

If both (2.3) and (2.4) are satisfied for every $x \in X$, we say that $f$ is the epi-limit of the sequence $f_{\nu}$ and we write

$$
f=\lim _{\nu} f_{\nu} .
$$

Note that in the presence of (2.3), in condition (2.4) we may replace the lim sup by limit. It can be shown that if the sequence $\left\{f_{\nu}, \nu=1, \ldots\right\}$ epi-convergences to $f$ at $x$, then necessarily $f$ is l.s.c. at $x$. In particular, it turns out that if $f_{\nu}=f$ for all $\nu$, the epi-limit function is $\mathrm{cl} f$, i.e.

$$
\lim _{\nu} f_{\nu}=\operatorname{cl} f .
$$

The study of epi-convergence has been motivated by the following results.

2.5 TheOREM. Suppose $(X, \tau)$ is metrizable, $f=\lim _{e} f_{\nu}$ and $\left\{x_{k}, k=1, \ldots\right\}$ is a sequence of points converging to $x$ such that $x_{k} \in \arg \min f_{\nu_{k}}$, i.e. $f_{\nu_{k}}\left(x_{k}\right)=\inf f_{\nu_{k}}$. Then $x \in \arg \min f$ and $\lim \left(\inf f_{\nu_{k}}\right)=\inf f$.

The proof will be omitted at this stage since this theorem is actually a corollary of a similar result that we obtain for sequences of saddle functions, cf. Theorem 3.10. Another key result in the theory of epi-convergence, is the following compactness theorem.

2.6 TheOREM. Suppose $(X, \tau)$ is metrizable and separable. Then any sequence of functions $\left\{f_{\nu}: X \rightarrow \bar{R}, \nu=1, \ldots\right\}$ admits an epi-convergent subsequence.

Again we shall not prove this result at this point since it is a corollary to Theorem 6.1 (for saddle functions). The reader should be warned that here we have not excluded the possibility that this subsequence converges to the function $f \equiv+\infty$, a degenerate situation from an optimization veiwpoint since it corresponds to the "empty" problem. 
For conceptual, as well as theoretical purposes, it is also useful to approach epi-limits in the following more general framework: $(X, \tau)$ is an arbitrary topological space and $\left\{f_{\nu}, \nu \in(N, \mathcal{H})\right\}$ is a filtered collection of extended-real valued functions defined on $X, N$ is the index space and $\mathcal{H}$ is a filter on $N$. For example, $N$ is the set of natural numbers and $\mathcal{H}=\{H \subset N \mid N \backslash H$ is finite $\}$ or $N$ is a topological space and $\mathcal{H}$ if the neighborhood system of a point. The epi-limit inferior of the collection $\left\{f_{\nu}, \nu \in N\right\}$, denoted by $\tau$-li $f_{e} f_{\nu}$-or simply $\mathrm{li}_{e} f_{\nu}$ when no confusion is possible - is defined by

$$
\left(\operatorname{li}_{e} f_{\nu}\right)(x)=\sup _{V \in \mathscr{T}_{\tau}(x)} \sup _{H \in \mathcal{K}} \inf _{\nu \in H} \inf _{y \in V} f_{\nu}(y) .
$$

The epi-limit superior, denoted by $\tau$-ls $f_{\nu}$ or simply ls $f_{e} f_{\nu}$, is defined by

$$
\left(\mathrm{ls}_{e} f_{\nu}\right)(x)=\sup _{V \in \mathscr{R}_{\tau}(x)} \inf _{H \in \mathcal{K}} \sup _{\nu \in H} \inf _{y \in V} f_{\nu}(y) .
$$

By $\ddot{\mathbb{Q}}$ we denote the grill associated to a collection $\mathcal{Q}$ of subsets of $N$, i.e. the family of all subsets of $N$ that meet every set in $Q$. If $\mathbb{Q}$ is a filter then the grill of $\ddot{Q}$ is $Q$ itself. Some interchanges of inf and sup are justified by the following lemma.

2.9 LEMMA. Let $Q$ be a collection of subsets of a space $A$ and $\ddot{Q}$ its grill. Then for any collection of extended-real numbers $\left\{x_{a}, a \in A\right\}$, we have

$$
\sup _{H \in \mathfrak{Q}} \inf _{a \in H} x_{a}=\inf _{H \in \mathbb{Q}} \sup _{a \in H} x_{a} .
$$

Consequently, if the grill of $\ddot{\mathbb{Q}}$ is again $A$, then also

$$
\sup _{H \in \ddot{Q}} \inf _{a \in H} x_{a}=\inf _{H \in \mathbb{Q}} \sup _{a \in H} x_{a} .
$$

Proof. Let $\beta=\sup _{H \in \mathbb{Q}} \inf _{a \in H} x_{a}$ and $\alpha=\inf _{H \in \ddot{\mathbb{Q}}} \sup _{a \in H} x_{a}$.

We first show that $\beta \geqslant \alpha$. We assume that $\beta<+\infty$, otherwise the inequality is trivially satisfied. Given any $\beta^{\prime}>\beta$, by definition of $\beta$ we have that

$$
\beta^{\prime} \geqslant \inf _{a \in H} x_{a} \text { for all } H \in \mathbb{Q} \text {. }
$$

Hence, for any $H \in \mathbb{Q}$, there exists $a_{H} \in H$ such that $x_{a_{H}}<\beta^{\prime}$. Let

$$
H^{\prime}=\left\{a \in A \mid x_{a}<\beta^{\prime}\right\} .
$$

In view of the above, $H^{\prime} \in \ddot{\mathbb{Q}}$, since $H^{\prime} \cap H \neq \varnothing$ for all $H \in \mathbb{Q}$. And we get

$$
\alpha=\inf _{H \in \ddot{\ddot{Q}}} \sup _{a \in H} x_{a} \leqslant \sup _{a \in H^{\prime}} x_{a} \leqslant \beta^{\prime},
$$

from which it follows that $\alpha \leqslant \beta$, since the preceding inequalities imply that $\alpha \leqslant \beta^{\prime}$ for all $\beta^{\prime}>\beta$.

We show next the reverse inequality, namely that $\beta \leqslant \alpha$. We assume that $\alpha<+\infty$; otherwise the inequality is trivially satisfied. Take any $\alpha^{\prime}>\alpha$. Then by definition of $\alpha$, we know that there exists $H^{\prime} \in \ddot{\mathbb{Q}}$ such that for all $a \in H^{\prime}, x_{a}<\alpha^{\prime}$. Since $H^{\prime}$ meets every $H \in \mathbb{Q}$, it follows that for all $H \in \mathbb{Q}$, $\inf _{a \in H} x_{a}<\alpha^{\prime}$ and hence

$$
\beta=\sup _{H \in \mathbb{Q}} \inf _{a \in H} x_{a} \leqslant \alpha^{\prime}
$$

from which it follows that $\beta \leqslant \alpha$, since $\beta \leqslant \alpha^{\prime}$ for all $\alpha^{\prime}>\alpha$. 
This lemma allows us to re-express the epi-limit superior as follows.

$$
\left(\operatorname{ls}_{e} f_{\nu}\right)(x)=\sup _{V \in N_{\tau}(x)} \sup _{H \in \mathscr{H} \mathcal{C}} \inf _{\nu \in H} \inf _{y \in V} f_{\nu}(y) .
$$

Since $\mathcal{H} \subset \ddot{H}$, it follows directly that

$$
\operatorname{li}_{e} f_{\nu} \leqslant \operatorname{ls}_{e} f_{\nu} .
$$

A function $f$ is the epi-limit of the collection of functions $\left\{f_{\nu}, \nu \in N\right\}$, and we write $\lim _{e} f_{\nu}=f$, if

$$
\operatorname{li}_{e} f_{\nu}=f=\operatorname{ls}_{e} f_{\nu} .
$$

One can verify that if $(X, \tau)$ is metrizable and the $\left\{f_{\nu}, \nu \in N\right\}$ is a sequence of functions, then $f=\operatorname{li}_{e} f_{\nu}=\mathrm{ls}_{e} f_{\nu}$ if and only if conditions (2.3) and (2.4) are satisfied. This will be proved in the setting of saddle functions. The terminology epi-limit and epi-convergence comes from the following theorem that provides a geometric interpretation of this limiting process.

2.14 ThEOREM $[9,5]$. Let $\left\{f_{\nu}, \nu \in N\right\}$ be a filtered collection of extended-real valued functions whose domain is endowed with a topology $\tau$. Then both $\operatorname{li}_{e} f_{\nu}$ and $\operatorname{ls}_{e} f_{\nu}$ are $\tau$-lower semicontinuous, since we have that

$$
\text { epi li } f_{\nu}=\limsup _{\nu \in N} \text { epi } f_{\nu}=\bigcap_{H \in \mathcal{H}} \operatorname{cl}\left(\bigcup_{\nu \in H} \text { epi } f_{\nu}\right),
$$

and

$$
\operatorname{epils}_{e} f_{\nu}=\liminf _{\nu \in N} \text { epi } f_{\nu}=\bigcap_{H \in \ddot{H} C} \operatorname{cl}\left(\bigcup_{\nu \in H} \text { epi } f_{\nu}\right) .
$$

A proof of this theorem in this form is given in [7]. It could also be obtained as a corollary of Theorem 4.16 .

So far our review of epi-convergence has stressed its local character or its geometric properties. There is also a variational approach that we sketch out here below. Here, we limit ourselves to a restricted class of perturbations that lead to a very specific class of approximates. These are the Yosida approximates (with parameter $\lambda$ ) defined by

$$
f_{\lambda}(x)=\inf _{u \in X}\left[f(u)+\frac{1}{2 \lambda} d_{\tau}^{2}(u, x)\right]
$$

where $d_{\tau}$ is a metric compatible with the topology $\tau$. We naturally assume that $(X, \tau)$ is metrizable. In $\S 5$, we show that the epi-convergence of a sequence of functions is intimately related to the convergence of the associated Yosida approximates; see Theorem 5.37. This was known for sequences of convex functions [1], but not for sequences of arbitrary functions.

Our emphasis will be on perturbations of the Yosida type. Our development will demonstrate that they provide us with a powerful and flexible theoretical tool. However, one should keep in mind that they are not the only type of perturbations that are of interest or that could be used to achieve the same objectives. For example in (2.17) we could replace $d_{\tau}^{2}$ by any function of $d_{\tau}$ which is locally Lipschitz and 
possesses some "shape" properties; cf. $\S 6$ of [13] about cast of functions. We shall return to this in $\S 5$.

For easy reference, we record here the hypograph version of the preceding results. A function $f$ from $X$ to $\bar{R}$ is said to be $\tau$-upper semicontinuous $(\tau$-u.s.c.) if hypo $f$ is closed, or equivalently if $f=$ (upper) cl $f$ where the upper closure of $f$ is the function cl $f$ such that hypo cl $f=\operatorname{cl}$ hypo $f$ or equivalently if

$$
f(x)=\inf _{x \in \mathscr{N}_{\tau}(x)} \sup _{x^{\prime} \in X} f\left(x^{\prime}\right) .
$$

The extended upper closure of $f$ is the function $\overline{\mathrm{cl}} f$ defined by

$$
\overline{\mathrm{cl}} f= \begin{cases}\mathrm{cl} f & \text { if } \mathrm{cl} f<+\infty, \\ +\infty & \text { otherwise. }\end{cases}
$$

From the context it will always be clear that we mean lower or upper closure.

A sequence of functions $\left\{f_{\nu}, \nu=1, \ldots\right\}$ defined on the metrizable space $X$ and with values in the extended reals hypo-converges to $f$ at $x$ if

$$
\begin{aligned}
& \text { for every subsequence of functions }\left\{f_{\nu_{k}}, k=1, \ldots\right\} \text { and } \\
& \text { sequence of points }\left\{x_{k}, k=1, \ldots\right\} \text { converging to } x \text {, we have } \\
& \text { that } \limsup _{k \rightarrow \infty} f_{\nu_{k}}\left(x_{k}\right) \leqslant f(x) \text { and } \\
& \text { there exists a sequence converging to } x \text { such that } \\
& \liminf _{\nu \rightarrow \infty} f_{\nu}\left(x_{\nu}\right) \geqslant f(x) \text {. }
\end{aligned}
$$

In general, given any collection of functions $\left\{f_{\nu}: X \rightarrow \bar{R}, \nu \in N\right\}$ filtered by $\mathcal{H}$, its hypo-limits interior and superior are defined by

$$
\left(\operatorname{li}_{h} f_{\nu}\right)(x)=\inf _{V \in \mathscr{N}_{\tau}(x)} \sup _{H \in \mathscr{K}} \inf _{\nu \in H} \sup _{y \in V} f_{\nu}(y),
$$

and

$$
\left(\operatorname{ls}_{h} f_{\nu}\right)(x)=\inf _{V \in \mathscr{N}_{\tau}(x)} \inf _{H \in \mathscr{K}} \sup _{\nu \in H} \sup _{y \in V} f_{\nu}(y) .
$$

The hypo-limit $\lim _{h} f_{\nu}$ exists if $\operatorname{li}_{h} f_{\nu}=\operatorname{ls}_{h} f_{\nu}=\lim _{h} f_{\nu}$. From Theorem 2.14 and Lemma 2.9 it follows that

$$
\operatorname{hypoli}_{h} f_{\nu}=\liminf _{\nu \in N} \operatorname{hypo} f_{\nu}=\bigcap_{H \in \ddot{\mathcal{H}}} \operatorname{cl}\left(\bigcup_{\nu \in H} \operatorname{hypo} f_{\nu}\right)
$$

and

$$
\text { hypo } \mathrm{s}_{h} f_{\nu}=\limsup _{\nu \in N} \text { hypo } f_{\nu}=\bigcap_{H \in \mathcal{H}} \operatorname{cl}\left(\bigcup_{\nu \in H} \operatorname{hypo} f_{\nu}\right) \text {. }
$$

As indicated at the beginning of this section, all variational or compactness results can be reformulated to fit the hypograph setting. For instance, Theorem 2.5 becomes: Suppose $(X, \tau)$ is metrizable and separable, $f=\lim _{h} f_{\nu}, x=\lim _{k \rightarrow \infty} x_{\nu_{k}}$ and $f_{\nu_{k}}\left(x_{\nu_{k}}\right)=\sup f_{\nu_{k}}$. Then $x$ maximizes $f$ and $\lim \left(\sup f_{\nu_{k}}\right)=\sup f$.

3. Epi/hypo-convergence. The metrizable case. We now consider saddle functions defined on $X \times Y$ with values in $\bar{R}$, the extended reals. Topologies on $X$ will be denoted by $\tau$, whereas we reserve $\sigma$ for the topologies on $Y$. In this section it is 
assumed that all topologies admit locally a countable base. Since in all the applications we can envisage, the (functional) spaces have closed point-sets and are regular, one may be tempted-and we shall succumb - to work with the more concrete assumption that all topologies are metrizable (without any real loss of generality). Let $F: X \times Y \rightarrow \bar{R}$ denote a generic saddle function, its effective domain is

$$
\operatorname{dom} F=\{(x, y) \in X \times Y \mid F(x, y) \in R\} .
$$

We associate with $F$ two multifunctions, each of which determine $F$ uniquely. They play the roles of the epigraph and of the hypograph associated with univariate functions. First the epigraph-multifunction

$$
y \mapsto \text { epi } F(\cdot, y)=\{(x, \alpha) \mid F(x, y) \leqslant \alpha\}
$$

which maps $Y$ into (epigraphical) subsets of $X \times R$, and the hypograph-multifunction

$$
x \mapsto \text { hypo } F(x, \cdot)=\{(y, \beta) \mid F(x, y) \geqslant \beta\}
$$

which maps $X$ into (hypographical) subsets of $Y \times R$.

If the multifunction $y \mapsto$ epi $F(\cdot, y)$ is closed-valued, i.e. for every $y$ the epigraph of the function $x \mapsto F(x, y)$ is closed, we say that $F$ is lower semicontinuous in $x$. By $\operatorname{cl}_{x} F: X \times Y \rightarrow \bar{R}$, we designate the bivariate function such that for all $y \in Y$,

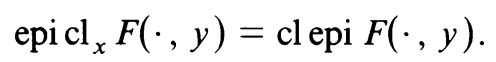

We call it the epi-closure of $F$. The lower closure of $F$, denoted by $\underline{\mathrm{cl}}_{x} F$, is such that for all $y \in Y$

$$
\underline{\mathrm{cl}}_{x} F(\cdot, y)= \begin{cases}\mathrm{cl}_{x} F(\cdot, y) & \text { if } \mathrm{cl}_{x} F(\cdot, y)>-\infty, \\ -\infty \text { on } X & \text { otherwise. }\end{cases}
$$

Similarly, $F$ is upper semicontinuous in $y$ if its hypo-closure $\operatorname{cl}_{y} F=F$, where $\mathrm{cl}_{y} F$ is the bivariate function from $X \times Y$ into $\bar{R}$ such that for all $x \in X$

$$
\text { hypo }_{y} F(x, \cdot)=\operatorname{cl}_{\text {hypo }} F(x, \cdot) \text {. }
$$

The upper closure of $F$, denoted by $\overline{\mathrm{c}}_{y} F$, is defined by

$$
\overline{c l}_{y} F(x, \cdot)= \begin{cases}\operatorname{cl}_{y} F(x, \cdot) & \text { if } \operatorname{cl}_{y} F(x, \cdot)<+\infty, \\ +\infty \text { on } X & \text { otherwise. }\end{cases}
$$

Typically, saddle functions take on both the values $+\infty$ and $-\infty$, and the lower and upper closure operations which in the univariate case dealt only with "degenerate" situations, arise naturally in the analysis of bivariate functions. This point has been stressed by Rockafellar in [10] in connection with his study of the convex-concave saddle functions. (Our terminology differs somewhat from his, because in the convex-concave case, in particular when seeking duality type results, it is convenient to work only with what we call the lower and upper closures of $F$, the use of extended closures lacks the flexibility that we like to preserve in the statements of our results.)

Let $\left\{F_{\nu}: X \times Y \rightarrow \bar{R}, \nu=1, \ldots\right\}$ be a sequence of bivariate functions, and both $(X, \tau)$ and $(Y, \sigma)$ be metrizable. We say that the sequence epi/hypo-converges to $F$ at $(x, y)$ if 
(3.6) for any subsequence of functions $\left\{F_{\nu_{k}}, k=1, \ldots\right\}$ and any sequence $\left\{x_{k}\right.$, $k=1, \ldots\} \tau$-converging to $x$, there exists a sequence $\left\{y_{k}, k=1, \ldots\right\} \sigma$-converging to $y$, such that

$$
F(x, y) \leqslant \liminf _{k \rightarrow \infty} F_{\nu_{k}}\left(x_{k}, y_{k}\right)
$$

and

(3.7) for any subsequence of functions $\left\{F_{\nu_{k}}, k=1, \ldots\right\}$ and any sequence $\left\{y_{k}\right.$, $k=1, \ldots\} \sigma$-converging to $y$, there exists a sequence $\left\{x_{k}, k=1, \ldots\right\} \tau$-converging to $x$ such that

$$
\limsup _{k \rightarrow \infty} F_{\nu_{k}}\left(x_{k}, y_{k}\right) \leqslant F(x, y) \text {. }
$$

If (3.6) and (3.7) are satisfied for all $(x, y)$, we say that the $F_{\nu}$ epi/hypo-converge to $F$. One should observe that epi/hypo-convergence is essentially a local property: in view of the definition, if the $F_{\nu}$ epi/hypo-converge to $F$, their restriction to some open set $\theta$ epi/hypo-converge to the restriction of $F$ to $\theta$.

When the functions $F_{\nu}$ and $F$ do not depend on $y$, then conditions (3.6) and (3.7) are clearly equivalent to (2.3) and (2.4) respectively, since for any sequence $\left\{a_{\nu} \in \bar{R}\right.$, $\nu=1, \ldots\}$ of extended-real numbers $\limsup _{\nu \rightarrow \infty} a_{\nu} \leqslant \alpha$ if and only if for every subsequence $\left\{a_{\nu_{k}}, k=1, \ldots\right\}$, lim $\sup _{k \rightarrow \infty} a_{\nu_{k}} \leqslant \alpha$. Similarly, if the $F_{\nu}$ and $F$ do not depend on $x$, these conditions are satisfied if and only if the sequence of functions $\left\{F_{\nu}, \nu=1, \ldots\right\}$ hypo-converges to $F$. Our results are thus bound to include those for univariate functions. However, one should not be lured into believing that epi/hypo-convergence is just the combination of the epi-convergence for each $y$ of the functions $x \mapsto F_{\nu}(x, y)$ with the hypo-convergence for each $x$ of the functions $y \mapsto F_{\nu}(x, y)$. We shall see later that it actually is a combination of epi- and hypo-convergence but of a much more sophisticated type. We record, however, the following sufficient condition for epi/hypo-convergence which is convenient in many applications (cf. Corollary 4.39).

3.8 Proposition. Suppose $(X, \tau)$ and $(Y, \sigma)$ are metrizable and $\left\{F_{\nu}: X \times Y \rightarrow \bar{R}\right.$, $\nu=1, \ldots\}$ is a sequence of saddle functions which satisfies: For every $y \in Y$, there exists a sequence $\left\{y_{\nu}, \nu=1, \ldots\right\}$-converging to $y$ such that the sequence $\left\{F_{\nu}\left(\cdot, y_{\nu}\right)\right.$ : $X \rightarrow \bar{R}, \nu=1, \ldots\}$ epi-converges to $F(\cdot, y)$, and similarly for every $x \in X$ there exists a sequence $\left\{x_{\nu}, \nu=1, \ldots\right\}$-converging to $x$ such that the sequence $\left\{F_{\nu}\left(x_{\nu}, \cdot\right)\right.$ : $Y \rightarrow \bar{R}, \nu=1, \ldots\}$ hypo-converges to $F(x, \cdot)$. Then, the sequence of saddle functions $F_{\nu}$ epi/hypo-converges to $F$.

The role played by epi/hypo-convergence in optimization and variational problems is underscored by the theorem that follows. Recall that $(\bar{x}, \bar{y})$ is a saddle point of the (bivariate) function $F$ if for all $x \in X$ and $y \in Y$ we have that

$$
F(\bar{x}, y) \leqslant F(\bar{x}, \bar{y}) \leqslant F(x, \bar{y})
$$

which implies that

$$
F(\bar{x}, \bar{y})=\min _{x \in X} \max _{y \in Y} F(x, y)=\max _{x \in X} \min _{y \in Y} F(x, y)
$$


where we write min and max to indicate that the infimum and the supremum are actually attained.

3.10 TheOREM. Suppose $(X, \tau)$ and $(Y, \sigma)$ are both metrixable and $\left\{F_{\nu}, \nu=1, \ldots\right\}$ is a sequence of bivariate functions defined on $X \times Y$ with values in the extended reals, which epi/hypo-converges to a function $F$. Suppose, moreover, that there is some subsequence of functions $\left\{F_{\nu_{k}}, k=1, \ldots\right\}$ with saddle points $\left\{\left(\bar{x}_{k}, \bar{y}_{k}\right), k=1, \ldots\right\}$, i.e., for all $x \in X$ and $y \in Y$

$$
F_{\nu_{k}}\left(\bar{x}_{k}, y_{k}\right) \leqslant F_{\nu_{k}}\left(\bar{x}_{k}, \bar{y}_{k}\right) \leqslant F_{\nu_{k}}\left(x_{k}, \bar{y}_{k}\right) \text {, }
$$

such that the $\bar{x}_{k}$-converge to some $\bar{x}$ and the $\bar{y}_{k} \sigma$-converge to some $\bar{y}$. Then $(\bar{x}, \bar{y})$ is a saddle point of $F$, and $F(\bar{x}, \bar{y})=\lim _{k \rightarrow \infty} F_{\nu_{k}}\left(\bar{x}_{k}, \bar{y}_{k}\right)$.

Proof. We have to show that (3.9) is satisfied. The fact that $\left(\bar{x}_{k}, \bar{y}_{k}\right)$ is a saddle point of $F_{\nu_{k}}$ implies that

$$
\sup _{y \in Y} F_{\nu_{k}}\left(\bar{x}_{k}, y\right) \leqslant F_{\nu_{k}}\left(\bar{x}_{k}, \bar{y}_{k}\right) \leqslant \inf _{x \in X} F_{v_{k}}\left(x, \bar{y}_{k}\right) .
$$

Hence, given any $y$ and any sequence $\left\{y_{k}, k=1, \ldots\right\}$ converging to $y$ (with respect to the topology $\sigma$ ), we get

$$
\liminf _{k \rightarrow \infty} F_{\nu_{k}}\left(\bar{x}_{k}, y_{k}\right) \leqslant \liminf _{k \rightarrow \infty} F_{\nu_{k}}\left(\bar{x}_{k}, \bar{y}_{k}\right)=: \alpha .
$$

Similarly, given any $x$ and any sequence $\left\{x_{k}, k=1, \ldots\right\}$ converging to $x$ (with respect to the topology $\tau$ ), we get

$$
\beta:=\limsup _{k \rightarrow \infty} F_{\nu_{k}}\left(\bar{x}_{k}, \bar{y}_{k}\right) \leqslant \limsup _{k \rightarrow \infty} F_{\nu_{k}}\left(x_{k}, \bar{y}_{k}\right) .
$$

From these inequalities, it follows that

$$
\sup _{y_{k} \rightarrow y} \liminf _{k \rightarrow \infty} F_{\nu_{k}}\left(\bar{x}_{k}, y_{k}\right) \leqslant \alpha \leqslant \beta \leqslant \inf _{x_{k} \rightarrow x} \limsup _{k \rightarrow \infty} F_{\nu_{k}}\left(x_{k}, \bar{y}_{k}\right) .
$$

Since (3.6) imples that for all $y$

$$
F(\bar{x}, y) \leqslant \sup _{y_{k} \rightarrow y} \liminf _{k \rightarrow \infty} F_{\nu_{k}}\left(\bar{x}_{k}, y_{k}\right)
$$

and (3.7) implies that for all $x$

$$
\inf _{x_{k} \rightarrow x} \limsup _{k \rightarrow \infty} F_{v_{k}}\left(x_{k}, \bar{y}_{k}\right) \leqslant F(x, \bar{y})
$$

we obtain that for all $y \in Y$ and $x \in X$

$$
F(\bar{x}, y) \leqslant \alpha \leqslant \beta \leqslant F(x, \bar{y}) .
$$

From this the saddle point inequality (3.9) is obtained. Substituting $\bar{x}$ for $x$, we get that $F(\bar{x}, y) \leqslant F(\bar{x}, \bar{y})$ for all $y \in Y$, and substituting $\bar{y}$ for $y$ similarly yields that $F(\bar{x}, \bar{y}) \leqslant F(x, \bar{y})$ for all $x \in X$.

To see that $F(\bar{x}, \bar{y})=\lim _{k \rightarrow \infty} F_{\nu_{k}}\left(\bar{x}_{k}, \bar{y}_{k}\right)$, simply observe that given $\left\{\bar{x}_{k}, k=\right.$ $1, \ldots\}$ converging to $\bar{x}$ epi/hypo-convergence implies that there exists $\left\{y_{k}, k=1, \ldots\right\}$ converging to $\bar{y}$ such that

$$
F(\bar{x}, \bar{y}) \leqslant \liminf _{k \rightarrow \infty} F_{\nu_{k}}\left(\bar{x}_{k}, y_{k}\right) \leqslant \liminf _{k \rightarrow \infty} F_{\nu_{k}}\left(\bar{x}_{k}, \bar{y}_{k}\right)
$$


where the second inequality follows from the fact that $F_{\nu_{k}}\left(\bar{x}_{k}, y_{k}\right) \leqslant F_{\nu_{k}}\left(\bar{x}_{k}, \bar{y}_{k}\right)$. Similarly, we get from the definition of epi/hypo-convergence and the fact that the $\left(\bar{x}_{k}, \bar{y}_{k}\right)$ is a saddle point,

$$
F(\bar{x}, \bar{y}) \geqslant \limsup _{k \rightarrow \infty} F_{\nu_{k}}\left(\bar{x}_{k}, \bar{y}_{k}\right) .
$$

The assertion now follows from the two preceding inequalities.

Our next theorem gives sufficient conditions under which a saddle point of $F$ can be obtained as the limit of near-saddle points of a sequence of functions $\left\{F_{\nu}\right.$, $\nu=1, \ldots\}$ that epi/hypo-converges to $F$. Related results for epi-convergence are given in [2, Theorems 2 and 3].

3.12 Proposition. Suppose $(X, \tau)$ and $(Y, \sigma)$ are metrizable and $\left\{F_{\nu}: X \times Y \rightarrow \bar{R}\right.$, $\nu=1, \ldots\}$ is a sequence of saddle functions such that

(i) for every $x \in X$, there exists a sequence $\left\{x_{\nu}, \nu=1, \ldots\right\}$-converging to $x$ such that the sequence $\left\{F_{\nu}\left(x_{\nu}, \cdot\right), \nu=1, \ldots\right\}$ hypo-converges to $F(x, \cdot)$ and

$$
\lim _{\nu \rightarrow \infty} \sup _{y \in Y} F_{\nu}\left(x_{\nu}, y\right)=\sup _{y \in Y} F(x, y)
$$

and

(ii) for every $y \in Y$, there exists a sequence $\left\{y_{\nu}, \nu=1, \ldots\right\}$-converging to y such that the sequence $\left\{F_{\nu}\left(\cdot, y_{\nu}\right), \nu=1, \ldots\right\}$ epi-converges to $F(\cdot, y)$ and

$$
\lim _{\nu \rightarrow \infty} \inf _{x \in X} F_{\nu}\left(x, y_{\nu}\right)=\inf _{x \in X} F(x, y) \text {. }
$$

Then if $(\bar{x}, \bar{y})$ is a saddle point of $F$,

$$
(\bar{x}, \bar{y}) \in \bigcap_{\varepsilon>0} \liminf _{\nu \rightarrow \infty}\left\{\varepsilon \text {-saddle points of } F_{\nu}\right\}
$$

where $\left(x^{\varepsilon}, y^{\varepsilon}\right)$ is an $\varepsilon$-saddle point of $F_{\nu}$ if

$$
F_{\nu}\left(x^{\varepsilon}, y^{\varepsilon}\right) \geqslant \sup _{y \in Y} F_{\nu}\left(x^{\varepsilon}, y\right)-\varepsilon
$$

and

$$
F_{\nu}\left(x^{\varepsilon}, y^{\varepsilon}\right) \leqslant \inf _{x \in X} F_{\nu}\left(x, y^{\varepsilon}\right)+\varepsilon .
$$

Note that the assumptions are stronger than epi/hypo-convergence; see Proposition 3.8.

Proof. By assumption there exist sequences $\left\{\bar{x}_{\nu}, \nu=1, \ldots\right\}$ and $\left\{\bar{y}_{\nu}, \nu=1, \ldots\right\}$ converging to $\bar{x}$ and $\bar{y}$ respectively such that

$$
F(\cdot, \bar{y})=\lim _{\nu \rightarrow \infty} F\left(\cdot, \bar{y}_{\nu}\right) \text { and } \lim _{\nu \rightarrow \infty} \inf F_{\nu}\left(\cdot, \bar{y}_{\nu}\right)=\inf F(\cdot, \bar{y}),
$$

and

$$
F(\bar{x}, \cdot)=\lim _{\nu \rightarrow \infty} F_{\nu}\left(\bar{x}_{\nu}, \cdot\right) \text { and } \lim _{\nu \rightarrow \infty} \sup F_{\nu}\left(\bar{x}_{\nu}, \cdot\right)=\sup F(\bar{x}, \cdot)
$$

The proposition will be proved if we show that for all $\varepsilon>0$ there exists $\nu_{\varepsilon}$ such that for all $\nu \geqslant \nu_{\varepsilon}$

$$
F_{\nu}\left(\bar{x}_{\nu}, \bar{y}_{\nu}\right) \leqslant \inf _{x \in X} F_{\nu}\left(x, \bar{y}_{\nu}\right)+\varepsilon,
$$


and

$$
F_{\nu}\left(\bar{x}_{\nu}, \bar{y}_{\nu}\right) \geqslant \sup _{y \in Y} F_{\nu}\left(\bar{x}_{\nu}, y\right)-\varepsilon
$$

i.e. for $\nu$ sufficiently large $\left(\bar{x}_{\nu}, \bar{y}_{\nu}\right)$ is an $\varepsilon$-saddle point of $F_{\nu}$.

We argue by contradiction. Suppose (3.18) is not true. Then there exists $\varepsilon_{0}>0$ and a subsequence indexed by $\left\{\nu_{k}, k=1, \ldots\right\}$ such that

$$
F_{k}\left(\bar{x}_{k}, \bar{y}_{k}\right)<\sup _{y} F_{k}\left(\bar{x}_{k}, y\right)-\varepsilon_{0}
$$

where we write $F_{k}$ for $F_{\nu_{k}}, \bar{x}_{k}$ for $\bar{x}_{\nu_{k}}$ and $\bar{y}_{k}$ for $\bar{y}_{\nu_{k}}$. Taking lim inf on both sides, using (3.15) and the sequential definition of epi-convergence at $x$, in particular (2.3), we get

$$
F(\bar{x}, \bar{y}) \leqslant \liminf _{k \rightarrow \infty} F_{k}\left(\bar{x}_{k}, \bar{y}_{k}\right) \leqslant \sup F(\bar{x}, \cdot)-\varepsilon_{0} .
$$

But this contradicts (3.9) if $\sup F(\bar{x}, \cdot)$ is finite which must be satisfied since by assumption $(\bar{x}, \bar{y})$ is a saddle point.

If $F(\bar{x}, \bar{y})=+\infty$, or similarly if $F(\bar{x}, \bar{y})=-\infty$, one has to take an extended notion of no $\varepsilon$-saddle point, namely

$$
F_{\nu}\left(x^{\varepsilon}, y^{\varepsilon}\right) \geqslant \inf \left[\sup _{y \in Y} F_{\nu}\left(x^{\varepsilon}, y\right)-\varepsilon, \varepsilon^{-1}\right]
$$

and

$$
F_{\nu}\left(x^{\varepsilon}, y^{\alpha}\right) \leqslant \sup \left[\inf _{x \in X} F_{\nu}\left(x, y^{\varepsilon}\right)+\varepsilon,-\varepsilon^{-1}\right] .
$$

One argues similarly that (3.17) must be satisfied, relying this time on the convergence of the infima in (3.15), and the hypo-convergence in (3.16).

4. Epi/hypo-convergence. Limit functions. We will be interested in collections of saddle functions $\left\{F_{\nu}, \nu \in N\right\}$-usually sequences of functions-defined on the product space $X \times Y$ and with values in the extended reals. Topologies on $X$ are denoted by $\tau$, whereas we reserve the symbol $\sigma$ for the topologies on $Y$. A priori, we make no assumptions about these topologies. The index set $N$ is assumed to be filtered by $\mathcal{K}$, a filter. Recall that if $N$ is the set of natural numbers and $\mathcal{H}=\{H \subset$ $N \mid N \backslash H$ is finite $\}$, then the limit on $N$ with respect to $\mathcal{H}$ is precisely the (sequential) limit of the sequence of functions $\left\{F_{\nu}, \nu=1, \ldots\right\}$. In this case, one can think of $\mathcal{H}$ as the system of "neighborhoods of $\{\infty\}$ ". Another example: $N=X$ (or $Y$ ) and $\mathcal{H}=\mathfrak{N}_{\tau}(x)$ (or $\Re_{\sigma}(y)$ resp.), the neighborhood system of a point $x$ (or $y$ ). The grill associated to $\mathcal{H}$, denoted by $\ddot{\mathscr{K}}$, is the family of subsets of $N$ that meet every set in $\mathcal{H}$. For the preceding examples, we get

$$
\ddot{\mathcal{H}}=\{\text { all infinite countable subsets of the natural numbers }\}
$$

and

$$
\ddot{\mathscr{H}}=\{\text { all subsets } H \text { of } X \mid x \in \operatorname{cl} H\} .
$$


When $N$ is the set of natural numbers, a limit with respect to an element of $\ddot{K}$ corresponds to the choice of a subsequence. Note that if $\mathcal{H}$ is a filter then

$$
\mathscr{H} \subset \ddot{H},
$$

and if $\mathcal{Q}$ is any collection of sets such that $A_{1} \in \mathcal{Q}, A_{2} \supset A_{1}$ implies that $A_{2} \in \mathbb{Q}$, then

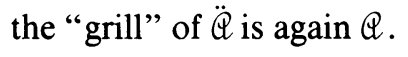

Thus, given a filtered collection of bivariate functions

$$
\left\{F_{\nu}: X \times Y \rightarrow \bar{R}, \nu=1, \ldots\right\},
$$

its $e$-limit, denoted by $l_{e} F_{\nu}$, is given by

$$
l_{e} F_{\nu}(x, y)=\sup _{U \in \mathscr{\Re}_{\tau}(x)} \inf _{V \in \mathscr{\Re}_{\sigma}(y)} \inf _{H \in \mathcal{H}} \sup _{\nu \in H} \sup _{v \in V} \inf _{u \in V} F_{\nu}(u, v) .
$$

Its $h$-limit, denoted by $l_{h} F_{\nu}$, is given by

$$
l_{h} F_{\nu}(x, y)=\inf _{V \in \mathscr{R}_{\sigma}(y)} \sup _{U \in \mathscr{T}_{\tau}(x)} \sup _{H \in \mathscr{H}} \inf _{\nu \in H} \inf _{u \in U} \sup _{v \in V} F_{\nu}(u, v) .
$$

The use of this terminology and symbolism is suggested by a number of results that follow. Observe that if the $F_{\nu}$ do not depend on $y$, then $l_{e} F_{\nu}$ is the epi-limit superior (2.8) of the sequence and $l_{h} F_{\nu}$ is its epi-limit inferior (2.7). Similarly, if the $F_{\nu}$ do not depend on $x$, then $l_{e} F_{\nu}$ is the hypo-limit inferior (2.22) of the sequence, whereas $l_{h} F_{\nu}$ is then its hypo-limit superior (2.23). Thus when the functions $F_{\nu}$ are actually univariate, there is an order relation between these two functions, cf. (2.12) for example. This is not the case in general: these two functions are not comparable. This is an important observation, in view of the definition that follows.

A (bivariate) function $F: X \times Y \rightarrow \bar{R}$ is an epi/hypo-limit of the sequence $\left\{F_{\nu}\right.$, $\nu=1, \ldots\}$ if

$$
F \leqslant l_{h} F_{\nu},
$$

and

$$
l_{e} F_{\nu} \leqslant F,
$$

and we then write that $F=\lim _{e / h} F_{\nu}$ and say that the filtered collection of saddle functions epi/hypo-converges to $F$. In general, an arbitrary collection of saddle functions does not admit an epi/hypo-limit, and if it does, the limit is not necessarily unique. To grasp this phenomena it is useful to consider a couple of examples.

4.7 Example. Take $X=Y=R$ and $N=\{1,2, \ldots\}$. For $\nu=1, \ldots$

$$
F_{1}=F_{\nu}(x, y)= \begin{cases}y^{x} & \text { if }(x, y) \in[0,1] \times[0,1] \text { with } 0^{0}=1, \\ -\infty & \text { if } x \in[0,1], y \notin[0,1], \\ +\infty & \text { otherwise. }\end{cases}
$$

Then $l_{h} F_{\nu}=F_{1}$ but

$$
l_{e} F_{\nu}(x, y)= \begin{cases}0 & \text { if }(x, y)=(0,0), \\ F_{1}(x, y) & \text { otherwise. }\end{cases}
$$

The epi/hypo-limit exists but is not unique. 
4.9 Example. Take $X=Y=R$ and $N=\{1,2, \ldots\}$. For $\nu$ odd

$$
F_{1}=F_{\nu}(x, y)= \begin{cases}y / x & \text { on }([0,1] \times[0,1]) \backslash\{0,0\} \\ 0 & \text { when } x=0, y=0, \\ -\infty & \text { if } x \in[0,1], y \notin[0,1], \\ +\infty & \text { otherwise, }\end{cases}
$$

and for $\nu$ even, $F_{2}=F_{\nu}(x, y)=2 F_{1}$. Then on $\left.\left.\left.] 0,1\right] \times\right] 0,1\right]$

$$
l_{h} F_{\nu}(x, y)=y / x<2 y / x=l_{e} F_{\nu}(x, y) \text {. }
$$

But $l_{h} F_{\nu}(0,0)=+\infty>l_{e} F_{\nu}(0,0)=0$. The limit functions are not comparable.

In $\S 3$, when $(X, \tau)$ and $(Y, \sigma)$ are metrizable and $\left\{F_{\nu}, \nu \in N\right\}$ is a sequence of saddle functions, we gave a sequential definition of epi/hypo-convergence. The proof that the sequential definition, and that via the limit functions $l_{e} F_{\nu}$ and $l_{h} F_{\nu}$ are the same, is surprisingly involved. It gives us a taste of the complexity of some of the arguments needed to obtain even the simplest facts about saddle functions.

4.10 TheOREM. Suppose $(X, \tau)$ and $(Y, \sigma)$ are metrizable and $\left\{F_{\nu}, \nu=1, \ldots\right\}$ is a sequence of saddle functions. Then

$$
l_{e} F_{\nu}(x, y)=\sup _{\substack{\left\{\nu_{k}, k=1, \ldots\right\} \\ y_{k} \rightarrow \sigma y}} \min _{\substack{x_{k} \rightarrow \tau x \\ \limsup _{k \rightarrow \infty}}} F_{\nu_{k}}\left(x_{k}, y_{k}\right),
$$

and

$$
l_{h} F_{\nu}(x, y)=\inf _{\substack{\left\{\nu_{k}, k=1, \ldots\right\} \subset N \\ x_{k} \rightarrow \tau x}} \max _{y_{k} \rightarrow \sigma y} \liminf _{k \rightarrow \infty} F_{\nu_{k}}\left(x_{k}, y_{k}\right)
$$

where the notation $\left\{v_{k}, k=1, \ldots\right\} \subset N$ means that we consider every possible subsequence of $\{1,2, \ldots\}=N$.

Proof. Since the definitions of $l_{e}$ and $l_{h}$ readily imply that $l_{e} F_{\nu}=-l_{h}\left(-F_{\nu}\right)$, it clearly suffices to prove either (4.11) or (4.12). We work with $l_{h} F_{\nu}$.

We show first that $l_{h} F_{\nu} \leqslant G$, where

$$
G(x, y)=\inf _{\substack{\left\{\nu_{k}\right\} \subset N \\ x_{k} \rightarrow \tau}} \sup _{y_{k} \rightarrow \sigma y} \liminf _{k \rightarrow \infty} F_{\nu_{k}}\left(x_{k}, y_{k}\right) .
$$

There is nothing to prove if $l_{h} F_{\nu}(x, y)=-\infty$. Let us thus assume that $l_{h} F_{\nu}(x, y)>$ $-\infty$. From the definition (4.4) of $l_{h} F_{\nu}$, we see that to any $\alpha<l_{h} F_{\nu}(x, y)$ and any $V \in \Re_{\sigma}(y)$ there corresponds a neighborhood $U$ of $x$ such that

$$
\alpha<\sup _{H \in \mathcal{H}} \inf _{\nu \in H} \inf _{u \in U} \sup _{v \in V} F_{\nu}(u, v)=\liminf _{\nu \rightarrow \infty} \inf _{u \in U} \sup _{v \in V} F_{\nu}(u, v) .
$$

Now let $\left\{V_{\mu}, \mu=1, \ldots\right\}$ be a countable base of (open) neighborhoods of $y$, decreasing with $\mu$ and such that $\cap_{\mu} V_{\mu}=\{y\}$. In view of our preceding remark, to each such $V_{\mu}$ corresponds $U_{\mu} \in \mathfrak{X}_{\tau}(x)$ such that

$$
\alpha<\liminf _{\nu \rightarrow \infty} \inf _{u \in U_{\mu}}\left[\sup _{v \in V_{\mu}} F_{\nu}(u, v)\right]
$$


and since for any $\left\{\nu_{k}, k=1, \ldots\right\} \subset N$, we have that $\liminf _{\nu \rightarrow \infty} \leqslant \liminf _{\nu_{k} \rightarrow \infty}$, we get

$$
\alpha<\liminf _{k \rightarrow \infty} \inf _{u \in U_{\mu}}\left[\sup _{v \in V_{\mu}} F_{k}(u, v)\right] \text { for all } \mu=1, \ldots
$$

Take any sequence $\left\{x_{k}, k=1, \ldots\right\}$ converging to $x$. Given any $U_{\mu}, x_{k} \in U_{\mu}$ for $k$ sufficiently large and thus

$$
\alpha<\liminf _{k \rightarrow \infty} \sup _{v \in V_{\mu}} F_{\nu_{k}}\left(x_{k}, v\right) .
$$

From Lemma A.5 (cf. Appendix) for $\mu=1, \ldots$ we can find $y_{k, \mu} \in V_{\mu}$ such that $\alpha<\liminf _{k \rightarrow \infty} F_{\nu_{k}}\left(x_{k}, y_{k, \mu}\right)$ and thus also

$$
\alpha \leqslant \liminf _{\mu \rightarrow \infty} \liminf _{k \rightarrow \infty} F_{\nu_{k}}\left(x_{k}, y_{k, \mu}\right) \text {. }
$$

We now have recourse to the diagonalization Lemma A.1, to extract a sequence $\left\{y_{k}=y_{k, \mu(k)}, k=1, \ldots\right\}$ such that $\alpha \leqslant \liminf _{k \rightarrow \infty} F_{\nu_{k}}\left(x_{k}, y_{k}\right)$ and since we can choose the $\mu(k)$ increasing with $k$, it follows that the sequence $\left\{y_{k}, k=1, \ldots\right\}$ $\sigma$-converges to $y$. Since the above inequality holds for any subsequence of functions $\left\{F_{\nu_{k}}, k=1, \ldots\right\}$, and any sequence $\left\{x_{k}, k=1, \ldots\right\} \tau$-converging to $x$, we have that

$$
\alpha \leqslant \inf _{\substack{\left\{\nu_{k}\right\} \subset N \\ x_{k} \rightarrow \tau x}} \sup _{y_{k} \rightarrow \sigma y} \liminf _{k \rightarrow \infty} F_{\nu_{k}}\left(x_{k}, y_{k}\right)=G(x, y) .
$$

Next we show that $G \leqslant l_{h} F_{\nu}$. Again there is nothing to prove if $l_{h} F_{\nu}(x, y)=+\infty$. Let us thus assume that $l_{h} F_{\nu}(x, y)<+\infty$. Given any $\beta>l_{h} F_{\nu}(x, y)$, the definition of $l_{h} F_{\nu}$ shows that we can find $V_{0} \in \mathfrak{N}_{\sigma}(y)$ such that for all $U \in \mathfrak{K}_{\tau}(x)$

$$
\beta>\sup _{H \in \mathcal{X}} \inf _{\nu \in H} \inf _{u \in U} \sup _{v \in V_{0}} F_{\nu}(u, v)=\liminf _{\nu \rightarrow \infty} \inf _{u \in U} \sup _{v \in V_{0}} F_{\nu}(u, v) \text {. }
$$

Let $\left\{U_{\mu}, \mu=1, \ldots\right\}$ be a countable base of (open) neighborhoods of $x$, decreasing with $\mu$ such that $\cap_{\mu} U_{\mu}=\{x\}$. From the above it follows that for all $\mu=1, \ldots$

$$
\beta>\liminf _{\nu \rightarrow \infty} \inf _{u \in U_{\mu}} \sup _{v \in V_{0}} F_{\nu}(u, v) .
$$

We can find $\left\{x_{\nu, \mu} \in U_{\mu}, \nu=1, \ldots\right\}$-cf. Corollary A.8 (Appendix)-such that for all $\mu$

$$
\beta>\liminf _{\nu \rightarrow \infty} \sup _{v \in V_{0}} F_{\nu}\left(x_{\nu, \mu}, v\right)
$$

and consequently

$$
\beta \geqslant \limsup _{\mu \rightarrow \infty} \liminf _{\nu \rightarrow \infty} \sup _{v \in V_{0}} F_{\nu}\left(x_{\nu, \mu}, v\right) .
$$

A standard diagonalization argument shows that there exists a choice $\left\{\nu_{\mu}, \mu=\right.$ $1, \ldots\}$ with $\nu_{\mu}$ increasing such that

$$
\beta \geqslant \limsup _{\mu \rightarrow \infty} \sup _{v \in V_{0}} F_{\nu_{\mu}}\left(x_{\mu}, v\right)
$$

where we have written $x_{\mu}$ for $x_{\nu}$. Now any sequence $\left\{y_{\mu}, \mu=1, \ldots\right\} \sigma$-converging to $y$ eventually belongs to $V_{0}$. Hence $\beta \geqslant \lim \sup _{\mu \rightarrow \infty} F_{\nu_{\mu}}\left(x_{\mu}, y_{\mu}\right)$. Since this holds for 
any sequence $\left\{y_{\mu}, \mu=1, \ldots\right\}$ converging to $y$ we get

$$
\beta \geqslant \sup _{y_{\mu} \rightarrow y} \limsup _{\mu \rightarrow \infty} F_{\nu_{\mu}}\left(x_{\mu}, y_{\mu}\right) \geqslant G(x, y)
$$

where the second inequality follows directly from the definition of $G$.

To complete the proof there remains only to show that in (4.13) $\sup _{y_{k} \rightarrow a y}$ is actually attained and thus the supremum is a maximum. Fix any subsequence $\left\{\boldsymbol{v}_{k}\right.$, $k=1, \ldots\} \subset N$ and $\left\{x_{k}, k=1, \ldots\right\} \tau$-convergent to $x$. Set

$$
a=\sup _{y_{k} \rightarrow y} \liminf _{k \rightarrow \infty} F_{\nu_{k}}\left(x_{k}, y_{k}\right)
$$

and let us first assume that $a<\infty$. This implies that for every $\varepsilon>0$, there exists $\left\{y_{k}^{\varepsilon}, k=1, \ldots\right\} \sigma$-convergent to $y$ such that

$$
\liminf _{k \rightarrow \infty} F_{\nu_{k}}\left(x_{k}, y_{k}^{\varepsilon}\right) \geqslant a-\varepsilon .
$$

Taking lim inf with respect to $\varepsilon \downarrow 0$, yields

$$
\liminf _{\varepsilon \downarrow 0} \liminf _{k \rightarrow \infty} F_{\nu_{k}}\left(x_{k}, y_{k}^{\varepsilon}\right) \geqslant a .
$$

We can find a sequence $\left\{y_{k}=y_{k}^{\varepsilon(k)}, k=1, \ldots\right\} \sigma$-convergent to $y$, see Lemma A.l, such that $\liminf _{k \rightarrow \infty} F_{v_{k}}\left(x_{k}, y_{k}\right) \geqslant a$ and clearly this is the sought-for sequence that achieves the maximum. Finally if $a=\infty$, then to every $n=1, \ldots$ there corresponds $\left\{y_{k}^{n}, k=1, \ldots\right\} \sigma$-convergent to $y$ such that $\liminf _{k \rightarrow \infty} F_{\nu_{k}}\left(x_{k}, y_{k}^{n}\right) \geqslant n$. Hence

$$
\liminf _{n \rightarrow \infty} \liminf _{k \rightarrow \infty} F_{\nu_{k}}\left(x_{k}, y_{k}^{n}\right)=\infty
$$

and again the diagonalization Lemma $\mathrm{A} .1$ yields the desired sequence $\left\{y_{k}, k=1, \ldots\right\}$ o-converging to $y$ such that $\liminf _{k \rightarrow \infty} F_{\nu_{k}}\left(x_{k}, y_{k}\right)=\infty$.

4.14 Corollary. Suppose $(X, \tau)$ and $(Y, \sigma)$ are metrizable and $\left\{F_{\nu}, \nu=1, \ldots\right\}$ is a sequence of saddle functions. Then

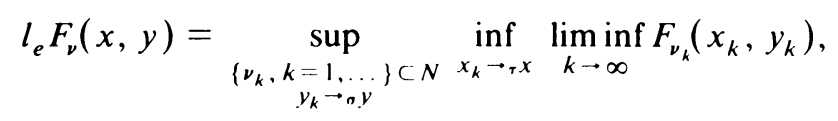

and

$$
l_{h} F_{\nu}(x, y)=\inf _{\substack{\left.\nu_{k}, k=1, \ldots\right\} \subset N \\ x_{k} \rightarrow \tau x}} \sup _{y_{k} \rightarrow \sigma, y} \limsup _{k \rightarrow \infty} F_{\nu_{k}}\left(x_{k}, y_{k}\right),
$$

where the notation $\left\{\nu_{k}, k=1, \ldots\right\} \subset N$ means that we consider all possible subsequences of $N=\{1,2, \ldots\}$.

Proof. The expressions (4.15) and (4.16) are precisely (4.11) and (4.12) except for the $\liminf _{\nu \rightarrow \infty}$ being replaced by limsup $\operatorname{sun}_{\nu \rightarrow \infty}$ and vice-versa. As in the proof of Theorem 4.10, we prove only the first one of these two identities, the other following then from the fact that $l_{h} F_{\nu}=-l_{e}\left(-F_{\nu}\right)$. In view of (4.11), it is immediate that

$$
l_{e} F_{\nu}(x, y) \geqslant \sup _{\substack{\left\{\nu_{k}, k=1, \ldots\right\} \subset N \\ y_{k} \rightarrow \sigma y}} \inf _{x_{k} \rightarrow+x} \liminf _{\nu \rightarrow \infty} F_{\nu_{k}}\left(x_{k}, y_{k}\right) .
$$


To obtain the converse, we note that for any subsequence of indices $\left\{\nu_{k}, k=1, \ldots\right\}$, any sequence $\left\{y_{k}, k=1, \ldots\right\} \sigma$-converging to $y$ and any sequence $\left\{x_{k}, k=1, \ldots\right\}$ $\tau$-converging to $x$, there corresponds a subsequence $\left\{\nu_{k p}, p=1, \ldots\right\}$ such that

$$
\limsup _{k \rightarrow \infty} F_{v_{k}}\left(x_{k}, y_{k}\right)=\lim _{p \rightarrow \infty} F_{v_{k p}}\left(x_{k p}, y_{k p}\right) \leqslant \liminf _{p \rightarrow \infty} F_{v_{k p}}\left(x_{k p}, y_{k p}\right) \text {, }
$$

as follows from the definition of lim inf and lim sup. We now use this in combination with (4.11) to obtain the desired inequality.

The $\tau$-lower semicontinuity of the function $x \mapsto l_{e} F_{\nu}(x, y)$ follows from the definition of $l_{e} F_{\nu}$ via (2.1) - cf. also Lemma 4.30 below-and similarly the $\sigma$-upper semicontinuity of $y \mapsto l_{h} F_{\nu}(x, y)$ follows from the definition of $l_{h} F_{\nu}$ via (2.18). We obtain these continuity properties as a consequence of the next theorem which shows that for all $y$, epi $l_{e} F_{\nu}(\cdot, y)$ is indeed the (set-) limit (with respect to both $\nu$ and $y$ ) of epigraphs, and for all $x$, hypo $l_{h} F_{\nu}(x, \cdot)$ is the limit of hypographs, the limit involving both $x$ and $\nu$.

4.17 THEOREM. Suppose $\left\{F_{\nu}, \nu \in N\right\}$ is a filtered collection of saddle functions defined on the product of the topological spaces $(X, \tau)$ and $(Y, \sigma)$. Then for all $y \in Y$,

$$
\text { epi } l_{e} F_{\nu}(\cdot, y)=\bigcap_{D \in \mathscr{D}_{y}} \operatorname{cl}\left(\bigcup_{\left(\nu, y^{\prime}\right) \in D} \text { epi } F_{\nu}\left(\cdot, y^{\prime}\right)\right)
$$

where $\mathscr{D}_{y}$ is the filter generated by $\mathcal{H} \times \mathscr{K}_{\sigma}(y)$, i.e. epi $l_{e} F_{\nu}(\cdot, y)$ is the liminf of the collection of sets $\left\{\right.$ epi $\left.F_{\nu}\left(\cdot, y^{\prime}\right) \mid \nu=1, \ldots, y^{\prime} \in Y\right\}$ with the index space $N \times Y$ filtered by $\mathscr{Q}_{y}$. Similarly for all $x \in X$

$$
\operatorname{hypo} l_{n} F_{\eta}(x, \cdot)=\bigcap_{D \in \mathscr{D}_{x}} \operatorname{cl}\left(\bigcup_{\left(\nu, x^{\prime}\right) \in D} \operatorname{hypo} F_{\nu}\left(x^{\prime}, \cdot\right)\right)
$$

where $\mathscr{Q}_{x}$ is the filter generated by $\mathcal{H} \times \mathscr{T}_{\tau}(x)$, i.e. hypo $l_{h} F_{\nu}(x, \cdot)$ is the lim inf of the collection of sets $\left\{\right.$ hypo $\left.F_{\nu}\left(x^{\prime}, \cdot\right) \mid \nu=1, \ldots, x^{\prime} \in X\right\}$ with the index space $N \times X$ filtered by $\mathscr{Q}_{x}$.

Proof. For reasons of symmetry, it clearly suffices to prove any one of these two identities. We derive (4.18). A pair

$$
(x, \alpha) \in \bigcap_{D \in \ddot{D}_{y}} \operatorname{cl}\left(\bigcup_{\left(\nu, y^{\prime}\right) \in D} \text { epi } F \nu\left(\cdot, y^{\prime}\right)\right)
$$

if and only if, for any $D \in \ddot{\mathscr{D}}_{y}$, any $U \in \Re_{\tau}(x)$, and any $\varepsilon>0$, there exists $\left(\nu, y^{\prime}\right) \in D$ such that

$$
U \times]-\infty, \alpha+\varepsilon\left[\cap \text { epi } F_{\nu}\left(\cdot, y^{\prime}\right) \neq \varnothing,\right.
$$

or equivalently if there exists $x^{\prime} \in U$ such that $F_{\nu}\left(x^{\prime}, y^{\prime}\right)<\alpha+\varepsilon$. But this holds if and only if

$$
\sup _{U \in \mathfrak{\Re}_{\tau}(x)} \sup _{D \in \ddot{\mathscr{D}}_{y}} \inf _{\left(\nu, y^{\prime}\right) \in D} \inf _{x^{\prime} \in U} F_{\nu}\left(x^{\prime}, y^{\prime}\right) \leqslant \alpha .
$$


Since $\mathscr{D}_{y}$ is a filter, the grill of $\ddot{\mathscr{D}}_{y}$ is $\mathscr{Q}_{y}$ and thus we can apply Lemma 2.9 to re-express this as follows:

$$
\sup _{U \in \mathcal{R}_{\tau}(x)} \inf _{D \in \mathcal{Q}_{y}} \sup _{\left(\nu, y^{\prime}\right) \in D} \inf _{x^{\prime} \in U} F_{\nu}\left(x^{\prime}, y^{\prime}\right) \leqslant \alpha .
$$

To complete the proof there remains only to show that

$$
\inf _{D \in \mathscr{P}_{y}} \sup _{\left(\nu, y^{\prime}\right) \in D}=\inf _{(H, V) \in \mathcal{H}^{\prime} \times \Re_{T}(y)} \sup _{\nu \in H, y^{\prime} \in V}
$$

because by substitution in the preceding inequality, we see that the left-hand side of (4.20) is nothing else than $l_{e} F_{\nu}(x, y)$, and $l_{e} F_{\nu}(x, y) \leqslant \alpha$ if and only if $(x, \alpha) \in$ epi $l_{e} F_{\nu}(\cdot, y)$.

Since $\mathcal{H} \times \mathscr{T}_{\tau}(y) \subset \mathscr{D}_{y}$, for any sequence of extended-real numbers doubly indexed by $\nu$ and $y^{\prime}$, say $\left\{a_{\nu, y^{\prime}} \mid \nu=1, \ldots, y^{\prime} \in Y\right\}$, we have that

$$
\inf _{D \in \mathscr{D}_{y}} \sup _{\left(\nu, y^{\prime}\right) \in D} a\left(\nu, y^{\prime}\right) \leqslant \inf _{(H, V) \in \mathscr{H}^{\prime} \times \Re_{\tau}(y)} \sup _{\nu \in H, y^{\prime} \in V} a\left(\nu, y^{\prime}\right),
$$

and thus to establish (4.21), it suffices to prove the converse. For any $D \in \mathscr{Q}_{y}$, there exists $H \in \mathcal{H}, V \in \mathfrak{N}_{\tau}(y)$ such that $H \times V \subset D$ and thus

$$
\sup _{\left(\nu, y^{\prime}\right) \in D} a\left(\nu, y^{\prime}\right) \geqslant \sup _{\nu \in H, y^{\prime} \in V} a\left(\nu, y^{\prime}\right) .
$$

From which it follows that

$$
\sup _{\left(\nu, y^{\prime}\right) \in D} a\left(\nu, y^{\prime}\right) \geqslant \inf _{(H, V) \in \mathcal{H}^{\prime} \times \mathfrak{R}_{\tau}(y)} \sup _{\nu \in H, y^{\prime} \in V} a\left(\nu, y^{\prime}\right) .
$$

Since this holds for every $D \in \mathscr{D}_{y}$, we also have that

$$
\inf _{D \in \mathscr{D}_{y}} \sup _{\left(\nu, y^{\prime}\right) \in D} a\left(\nu, y^{\prime}\right) \geqslant \inf _{(H, V) \in \mathcal{H} \times \Re_{\tau}(y)} \sup _{\nu \in N, y^{\prime} \in Y} a\left(\nu, y^{\prime}\right)
$$

which completes the argument.

It is important to realize that $\ddot{\mathscr{D}}_{y}$ is not equal to $\ddot{\mathcal{K}} \times \mathscr{\Re}_{\tau}(y)$. Naturally, one always has that $\ddot{\mathcal{C}} \times \ddot{\mathscr{N}}_{\tau}(y) \subset \ddot{\mathscr{D}}_{y}$ but in general $\ddot{\mathscr{D}}_{y}$ is a much richer collection of sets and we would not obtain the epigraph of $l_{e} F_{\nu}(\cdot, y)$ if in (4.18) we replaced $\ddot{\mathscr{D}}_{y}$ by $\ddot{\mathcal{K}} \times \ddot{\mathscr{H}}_{\tau}(y)$. As an immediate consequence of the representations (4.18) and (4.19) and the definition of upper and lower semicontinuity, we have

4.22 Corollary. Suppose $\left\{F_{\nu}, \nu \in N\right\}$ is a filtered collection of saddle functions defined on the product of the topological spaces $(X, \tau)$ and $(Y, \sigma)$. Then its e-limit $l_{e} F_{\nu}$ is $\tau$-l.s.c. in $x$ for all $y$, and its $h$-limit $l_{h} F_{\nu}$ is $\sigma$-u.s.c. in $y$ for all $x$.

Penalty methods and various approximation schemes depend on monotonically increasing or decreasing sequences of functions. For epi/hypo-convergence we have the following result.

4.23 Proposition. Suppose $\left\{F_{\nu}, \nu=1, \ldots\right\}$ is an increasing (or decreasing) sequence of saddle functions defined on the product of the topological spaces $(X, \tau)$ and $(Y, \tau)$. Then the sequence is epi/hypo-convergent. 
Proof. Again for reasons of symmetry, it is sufficient to consider the case of an increasing sequence. Given any pair $U \in \Re_{\tau}(x), V \in \Re_{\sigma}(y)$, the sequence

$$
\left\{\inf _{x^{\prime} \in U} \sup _{y^{\prime} \in V} F_{\nu}\left(x^{\prime}, y^{\prime}\right), \nu=1, \ldots\right\}
$$

is increasing. Thus

$$
\liminf _{\nu \rightarrow \infty} \inf _{x^{\prime} \in U} \sup _{y^{\prime} \in V} F_{\nu}\left(x^{\prime}, y^{\prime}\right)=\lim _{\nu \rightarrow \infty} \inf _{x^{\prime} \in U} \sup _{y^{\prime} \in V} F_{\nu}\left(x^{\prime}, y^{\prime}\right),
$$

and similarly

$$
\limsup _{\nu \rightarrow \infty} \sup _{y^{\prime} \in V} \inf _{x^{\prime} \in U} F_{\nu}\left(x^{\prime}, y^{\prime}\right)=\lim _{\nu \rightarrow \infty} \sup _{y^{\prime} \in V} \inf _{x^{\prime} \in U} F_{\nu}\left(x^{\prime}, y^{\prime}\right) .
$$

Together the inequalities imply that for all $U \in \mathfrak{N}_{\tau}(x)$ and $V \in \mathfrak{N}_{\sigma}(y)$

$$
\limsup \sup _{\nu \rightarrow \infty} \inf _{y^{\prime} \in V} F_{\nu}\left(x^{\prime}, y^{\prime}\right) \leqslant \liminf _{\nu \rightarrow \infty} \inf _{x^{\prime} \in U} \sup _{\substack{y^{\prime} \in V \\ y^{\prime} \in F^{\prime}}} F_{\nu}\left(x^{\prime}, y^{\prime}\right) \text {. }
$$

Since sup inf $\leqslant$ inf sup, we get

$$
\begin{aligned}
\sup _{U \in \Re_{\tau}(x)} \inf _{V \in \Re_{0}(y)} \limsup _{\nu \rightarrow \infty} \sup _{y^{\prime} \in V} \inf _{x^{\prime} U} F_{\nu}\left(x^{\prime}, y^{\prime}\right)=l_{e} F_{\nu}(x, y) \\
\quad \leqslant \inf _{V \in \Re_{0}(y)} \sup _{U \in \Re_{\tau}(x)} \liminf _{\nu \rightarrow \infty} \inf _{x^{\prime} \in U} \sup _{y^{\prime} \in V} F_{\nu}\left(x^{\prime}, y^{\prime}\right)=l_{h} F_{\nu}(x, y),
\end{aligned}
$$

which implies the epi/hypo-convergence of the $F_{\nu}$; see (4.5) and (4.6).

This proposition implies the epi-convergence of increasing and decreasing sequences of (univariate) functions, facts that are exploited repetitively in many applications of the theory, cf. for example the convergence proofs for barrier and penalty functions in [2].

To any (filtered) collection of saddle functions, we have, so far, associated a couple of limit functions designated by $l_{e} F_{\nu}$ and $l_{h} F_{\nu}$. The convergence of saddle points is intimately related to some inequalities involving these two functions. However there are many other limit functions. In [5] De Giorgi suggests a number of them. Considering all possible interchanges of the sequence of inf's and sup's that appear in (4.3) and (4.4) and possible substitutions of in $\dot{f}$ and sup, there are at least 180 limit functions that one could associate to a filtered collection of saddle functions. Of course, not all of them are interesting. However there are various relations between some of them that can be used to assert specific properties or to verify if the collection epi/hypo-converges. We consider the following 8 limit functions associated to the sequence of saddle functions $\left\{F_{\nu}, \nu=1, \ldots\right\}$,

$$
\begin{aligned}
& l_{1} F_{\nu}(x, y)=\inf _{v \in \Re_{\sigma}(y)} \sup _{U \in \mathcal{R}_{\tau}(x)} \limsup _{\nu \rightarrow \infty} \inf _{u \in U} \sup _{v \in V} F_{\nu}(u, v), \\
& l_{2} F_{\nu}(x, y)=\sup _{U \in \Re_{\tau}(x)} \inf _{V \in \Re_{o}(y)} \limsup _{\nu \rightarrow \infty} \inf _{u \in U} \sup _{v \in V} F_{\nu}(u, v), \\
& l_{3} F_{\nu}(x, y)=\inf _{v \in \mathfrak{R}_{\sigma}(y)} \sup _{U \in \mathfrak{\Re}_{\tau}(x)} \limsup _{\nu \rightarrow \infty} \sup _{v \in V} \inf _{u \in U} F_{\nu}(u, v), \\
& l_{e} F_{\nu}(x, y)=\sup _{U \in \Re_{\tau}(x)} \inf _{V \in \Re_{\sigma}(y)} \limsup _{\nu \rightarrow \infty} \sup _{v \in V} \inf _{u \in U} F_{\nu}(u, v), \\
& l_{h} F_{\nu}(x, y)=\inf _{V \in \Re_{\sigma}(y)} \sup U \in \Re_{\tau}(x) \liminf _{\nu \rightarrow \infty} \inf _{u \in U} \sup _{v \in V} F_{\nu}(u, v),
\end{aligned}
$$




$$
\begin{aligned}
& l_{4} F_{\nu}(x, y)=\inf _{V \in \Re_{\sigma}(y)} \sup _{U \in \Re_{\tau}(x)} \liminf _{\nu \rightarrow \infty} \sup _{v \in V} \inf _{u \in U} F_{\nu}(u, v), \\
& l_{5} F_{\nu}(x, y)=\sup _{U \in \mathcal{R}_{T}(x)} \inf _{V \in \mathscr{N}_{\sigma}(y)} \liminf _{\nu \rightarrow \infty} \inf _{u \in U} \sup _{v \in V} F_{\nu}(u, v), \\
& l_{6} F_{\nu}(x, y)=\sup _{U \in \mathscr{R}_{\tau}(x)} \inf _{v \in \mathscr{N}_{o}(y)} \liminf _{\nu \rightarrow \infty} \sup _{v \in V} \inf _{u \in U} F_{\nu}(u, v) .
\end{aligned}
$$

For the sake of completeness, we have rewritten here the expressions for $l_{e} F_{\nu}$ and $l_{h} F_{\nu}$. Rather than sequences of functions, we could have considered arbitrary filtered collections; one need only replace $\liminf _{\nu \rightarrow \infty}$ by $\sup _{H \in \mathcal{H}} \inf _{\nu \in H}$, and lim $\sup _{\nu \rightarrow \infty}$ by $\inf _{H \in \mathcal{K}} \sup _{\nu \in H}$. Using these facts that supinf $\leqslant \inf \sup$ and $\lim$ inf $\leqslant \lim \sup$, we obtain relations between these functions as shown in Figure 1 , where the $\cdots \leqslant \cdots$ indicate the order relations.

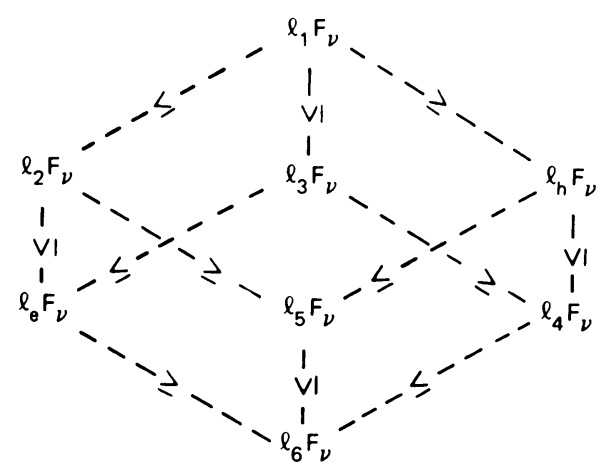

Figure 1. Order relations

The functions $l_{2} F_{\nu}, l_{5} F_{\nu}, l_{6} F_{\nu}$, and naturally $l_{e} F_{\nu}$ are $\tau$-l.s.c. in $x$, and the functions $l_{1} F_{\nu}, l_{3} F_{\nu}, l_{4} F_{\nu}$ as well as $l_{h} F_{\nu}$ are $\sigma$-l.s.c. in $y$. All of this can be directly obtained as a consequence of the definitions and the following easy lemma.

4.30 Lemma. Suppose $(X, \tau)$ is a topological space and $a(x)=\sup _{U \in \mathfrak{\vartheta}_{\tau}(x)} q(U)$, where $q$ is an extended-real valued function defined on the subsets of $X$. Then $a$ is $\tau$-lower semicontinuous.

Proof. We have to show that epi $a$ is closed, or equivalently that given any pair $(x, \alpha) \in X \times R$ such that

$$
\text { for all } \left.\varepsilon>0 \text { and } U \in \mathscr{T}_{\tau}(x), \quad U \times\right]-\infty, \alpha+\varepsilon[\cap \text { epi } a \neq \varnothing,
$$

it follows that $(x, \alpha) \in$ epi $a$, i.e. $\alpha \geqslant a(x)$. Suppose to the contrary that $\alpha<a(x)$. Then by definition of $a$, there exist $\varepsilon^{\prime}>0$ and $U^{\prime} \in \Re_{\tau}(x)$ such that $\alpha+\varepsilon^{\prime}<q\left(U^{\prime}\right)$. Denote by $U^{\prime \prime}$ the interior of $U^{\prime}$, and observe that for all $x^{\prime} \in U^{\prime \prime}, U^{\prime} \in \Re_{\tau}\left(x^{\prime}\right)$. Thus for all $x^{\prime} \in U^{\prime \prime}, a\left(x^{\prime}\right) \geqslant q\left(U^{\prime}\right)>\alpha+\varepsilon^{\prime}$. Hence

$$
\left.U^{\prime \prime} \times\right]-\infty, \alpha+\varepsilon^{\prime}[\cap \text { epi } a=\varnothing
$$

which contradicts the hypothesis, since $U^{\prime \prime} \in \mathfrak{N}_{\tau}(x)$.

Given a sequence of functions $\left\{F_{\nu}, \nu=1, \ldots\right\}$ any condition of the type $F \leqslant l_{i} F_{\nu}$ or /and $F \geqslant l_{j} F_{\nu}$ (with $i, j=1,2, \ldots, 6, e, h$ ) induces certain limit properties for the 
function $F$. The relationship between various types of convergences can be obtained from the diagram given by Figure 1. Each type of convergence possesses some interest in its own right. An independent study of each possible type would lead us too far astray from the main interest of this paper. At this time we limit ourselves to one other type of convergence, namely that induced by the conditions

$$
l_{1} F_{\nu} \leqslant F \leqslant l_{6} F_{\nu} \text {. }
$$

From Figure 1 we know that $l_{1} F_{\nu} \geqslant l_{6} F_{\nu}$. Thus if (4.31) is satisfied it means that all the limit functions that have been associated with the sequences $\left\{F_{\nu}, \nu=1, \ldots\right\}$ must be equal, in particular $l_{1} F_{\nu}=F=l_{6} F_{\nu}$. Moreover, in view of our remarks following Figure 1, the function $F$ must be both $\tau$-l.s.c. in $x$ and $\sigma$-l.s.c. in $y$. In terms of the applications we have in mind these would be very strong properties. In fact (4.31) induces a convergence for saddle functions that is much stronger than epi/hypo-convergence. This is substantiated by the results that follow.

4.32 Proposition. Suppose $\left\{F_{\nu}, \nu \in N\right\}$ is a filtered collection of saddle functions defined on the product of the topological spaces $(X, \tau)$ and $(Y, \sigma)$. Then for all $y \in Y$

$$
\text { epi } l_{6} F_{\nu}(\cdot, y)=\bigcap_{E \in \ddot{\mathcal{E}}_{y}} \mathrm{cl}\left(\bigcup_{(\nu, v) \in E} \operatorname{epi} F_{\nu}(\cdot, \nu)\right) \text {, }
$$

and for all $x \in X$

$$
\text { hypo } l_{1} F_{\nu}(x, \cdot)=\bigcap_{E \in \ddot{\mathscr{E}}_{x}} \operatorname{cl}\left(\bigcup_{(\nu, u) \in E} \operatorname{hypo} F_{\nu}(u, \cdot)\right),
$$

where $\varepsilon_{y}=\ddot{H} \times \mathscr{N}_{\sigma}(y)$ and $\varepsilon_{x}=\ddot{\mathscr{K}} \times \Re_{\sigma}(x)$.

It is worth noting that neither epi $l_{6} F_{\nu}(\cdot, y)$ nor hypo $l_{1} F_{\nu}(x, \cdot)$ are the liminf of epigraphs or hypographs with respect to some filter on $\Re \times \cdot$, as was the case for epi $l_{e} F_{\nu}(\cdot, y)$ and hypo $l_{h} F_{\nu}(x, \cdot)$. The family $\varepsilon_{y}$ (or $\varepsilon_{x}$ ) does not engender a filter because some finite subcollections have empty intersection.

Proof. Again for reasons of symmetry it suffices to prove any one of these two identities. We derive (4.33). A pair

$$
(x, \alpha) \in \bigcap_{E \in \ddot{\mathscr{E}}_{y}} \mathrm{cl} \bigcup_{(\nu, v) \in E} \operatorname{epi} F_{\nu}(\cdot, v)
$$

if and only if for all $E \in \ddot{\mathcal{E}}_{y}, U \in \Re_{\tau}(x)$ and $\varepsilon>0$, we can find $(\nu, v) \in E$ and $u \in U$ such that $F_{\nu}(u, v)<\alpha+\varepsilon$. Or equivalently, if and only if

$$
\sup _{U \in \Re_{\tau}(x)} \sup _{E \in \ddot{\mathscr{E}}_{y}} \inf _{(\nu, v) \in E} \inf _{u \in U} F_{\nu}(u, v) \leqslant \alpha,
$$

or still, in view of Lemma 2.9, if and only if

$$
\sup _{U \in \mathscr{T}_{\tau}(x)} \inf _{E \in \mathscr{E}_{y}^{\prime}} \sup _{(\nu, v) \in E} \inf _{u \in U} F_{\nu}(u, v) \leqslant \alpha
$$

where $\varepsilon_{y}^{\prime}=\left(\ddot{\varepsilon}_{y}\right)$ is the grill of $\ddot{\varepsilon}_{y}$. The proof will be complete if we can show that we can replace $\mathcal{E}_{y}^{\prime}$ by $\mathcal{E}_{y}$ in the preceding inequality, because then the term on the left 
is $l_{6} F_{\nu}(x, y)$ and $l_{6} F_{\nu}(x, y) \leqslant \alpha$ if and only if $(x, \alpha) \in$ epi $l_{6} F_{\nu}(\cdot, y)$. It thus remains only to show that for any collection of extended-real numbers $\left\{a_{\nu}(v) \mid \nu \in N\right.$, $v \in Y\}$

$$
\inf _{E \in \mathcal{E}_{y}^{\prime}} \sup _{(\nu, v) \in E} a_{\nu}(v)=\inf _{E \in \mathcal{E}_{y}} \sup _{(\nu, v) \in E} a_{\nu}(v)
$$

Since $\varepsilon_{y}^{\prime} \supset \mathcal{E}_{y}$ it clearly suffices to show that the $\geqslant$ (larger than or equal to) inequality holds. But now note that from the definition of the (double) grill of a collection of sets it follows that

$$
\mathcal{E}_{y}^{\prime}=\left\{E \subset N \times Y \mid \exists D \in \mathcal{E}_{y} \text { with } D \subset E\right\} .
$$

Thus given any set $E \in \mathcal{E}_{y}^{\prime}$ we can find $D \in \mathcal{E}_{y}$ such that $D \subset E$ and thus

$$
\sup _{(\nu, v) \in E} a_{\nu}(v) \geqslant \sup _{(\nu, v) \in D} a_{\nu}(v) \geqslant \inf _{D \in \mathcal{E}_{y}} \sup _{(\nu, v) \in D} a_{\nu}(v) .
$$

The desired inequality follows from the above since it holds for all $E$.

4.35 Proposition. Suppose $(X, \tau)$ and $(Y, \sigma)$ are metrizable, and $\left\{F_{\nu}: X \times Y \rightarrow \bar{R}\right.$, $\nu=1, \ldots\}$ is a sequence of saddle functions. Then

$$
\begin{aligned}
& l_{6} F_{\nu}(x, y)=\sup _{y_{\nu} \rightarrow \sigma y} \inf _{x_{\nu} \rightarrow \tau} \liminf _{\nu \rightarrow \infty} F_{\nu}\left(x_{\nu}, y_{\nu}\right) \\
& =\sup _{y_{\nu} \rightarrow 0 y} \inf _{\left\{\nu_{k}, k=1, \ldots\right\} \subset N} \liminf _{k \rightarrow \infty} F_{\nu_{k} \rightarrow \tau}\left(x_{k}, y_{\nu_{k}}\right) \\
& =\sup _{y_{\nu} \rightarrow 0 y} \inf _{\left\{v_{k}, k=1, \ldots\right\} \subset N} \limsup _{\substack{x_{k} \rightarrow \tau \\
x_{k} \rightarrow \infty}} F_{\nu_{k}}\left(x_{k}, y_{\nu_{k}}\right)
\end{aligned}
$$

and

$$
\begin{aligned}
& l_{1} F_{\nu}(x, y)=\inf _{x_{\nu} \rightarrow \tau x} \sup _{y_{\nu} \rightarrow \sigma y} \limsup _{\nu \rightarrow \infty} F_{\nu}\left(x_{\nu}, y_{\nu}\right) \\
& =\inf _{x_{\nu} \rightarrow \tau} \sup _{\substack{\left\{\nu_{k}, k=1, \ldots\right\} \\
y_{k} \rightarrow \sigma y}} \limsup _{\substack{k \rightarrow \infty \\
k \rightarrow \infty}} F_{\nu_{k}}\left(x_{\nu_{k}}, y_{k}\right) \\
& =\inf _{x_{\nu} \rightarrow \tau x} \sup _{\substack{\left\{\nu_{k}, k=1, \ldots\right\} \subset N \\
y_{k} \rightarrow \sigma y}} \liminf _{k \rightarrow \infty} F_{\nu_{k}}\left(x_{\nu_{k}}, y_{k}\right) \text {. }
\end{aligned}
$$

Proof. Once more for reasons of symmetry it will be sufficient to derive one of these two strings of equalities, we work with (4.36). The argument that justifies the last equalities is similar to that used to prove Corollary 4.14. The second expression is obtained from the first one by observing that clearly

$$
\inf _{x_{\nu} \rightarrow x} \liminf _{\nu \rightarrow \infty} F_{\nu} \geqslant \inf _{\substack{\left\{\nu_{k}\right\} \subset N \\ x_{k} \rightarrow x}} \liminf _{k \rightarrow \infty} F_{\nu_{k}}
$$

and if $\left\{\nu_{k}\right\} \subset N, x_{k} \rightarrow x$, then the sequence

$$
\begin{aligned}
& x_{\nu_{k}}=x_{k}, \quad k=1, \ldots, \\
& x_{\nu}=x, \quad \text { for all } \nu \neq \nu_{k},
\end{aligned}
$$


is such that

$$
\liminf _{\nu \rightarrow \infty} F_{\nu}\left(x_{\nu}, y_{\nu}\right) \leqslant \liminf _{k \rightarrow \infty} F_{\nu_{k}}\left(x_{k}, y_{\nu_{k}}\right)
$$

which yields the reverse inequality.

Next we show that $l_{6} F_{\nu}(x, y)=g(x, y)$ where

$$
g(x, y)=\sup _{y_{\nu} \rightarrow y} \inf _{x_{\nu} \rightarrow x} \liminf _{\nu \rightarrow \infty} F_{\nu}\left(x_{\nu}, y_{\nu}\right) .
$$

We start by proving that $l_{6} F_{\nu}(x, y) \leqslant g(x, y)$. There is nothing to prove if $l_{6} F_{\nu}(x, y)$ $=-\infty$, so let us assume that $l_{6} F_{\nu}(x, y)>-\infty$. Take any $\alpha<l_{6} F_{\nu}(x, y)$. From the definition (4.29) of $l_{6} F_{\nu}$, this implies that there exists $U \in \Re_{\tau}(x)$ such that for all $V \in \Re_{\sigma}(y)$

$$
\alpha<\liminf _{\nu \rightarrow \infty} \sup _{v \in V} \inf _{u \in U} F_{\nu}(u, v)
$$

Let $\left\{V_{m}, m=1, \ldots\right\}$ be a decreasing sequence of neighborhoods of $y$ such that $\cap_{m=1}^{\infty} V_{m}=\{y\}$. From the above and Lemma A.5 it follows that there exists $v_{\nu, m} \in V_{m}$ such that

$$
\alpha<\liminf _{\nu \rightarrow \infty} \inf _{u \in U} F_{\nu}\left(u, v_{\nu, m}\right)
$$

and hence

$$
\alpha \leqslant \liminf _{m \rightarrow \infty} \liminf _{\nu \rightarrow \infty} \inf _{u \in U} F_{\nu}\left(u, v_{\nu, m}\right) .
$$

The diagonalization Lemma A.1 (see the Appendix) allows us to extract a sequence $\left\{y_{\nu}=v_{\nu, m(\nu)}, \nu=1, \ldots\right\} \sigma$-convergent to $y$ such that

$$
\alpha \leqslant \liminf _{\nu \rightarrow \infty} \inf _{u \in U} F_{\nu}\left(u, y_{\nu}\right) \text {. }
$$

Take any sequence $\left\{x_{\nu}, \nu=1, \ldots\right\} \tau$-convergent to $x$. For $\nu$ sufficiently large $x_{\nu} \in U$ and hence $\alpha \leqslant \liminf F_{\nu}\left(x_{\nu}, y_{\nu}\right)$. Since this holds for any sequence $\left\{x_{\nu}, \nu=1, \ldots\right\}$ it follows that

$$
\alpha \leqslant \inf _{x_{\nu} \rightarrow \tau} \liminf _{\nu \rightarrow \infty} F_{\nu}\left(x_{\nu}, y_{\nu}\right)
$$

and a fortiori

$$
\alpha \leqslant \sup _{y_{\nu} \rightarrow \sigma y} \inf _{x_{\nu} \rightarrow \tau x} \liminf _{\nu \rightarrow \infty} F_{\nu}\left(x_{\nu}, y_{\nu}\right)=g(x, y)
$$

This holds for any $\alpha<l_{6} F_{\nu}(x, y)$, and thus $l_{6} F_{\nu} \leqslant g$.

Next we show that $l_{6} F_{\nu}(x, y) \geqslant g(x, y)$. The inequality is trivially satisfied if $l_{6} F_{\nu}(x, y)=+\infty$. Thus let us give the proof under the assumption that $l_{6} F_{\nu}(x, y)<$ $+\infty$. Take any $\beta>l_{6} F_{\nu}(x, y)$. This means that for any $U \in \Re_{\tau}(x)$, there exists $V \in \mathfrak{T}_{\sigma}(y)$ such that

$$
\beta>\liminf \sup _{\nu \rightarrow \infty} \inf _{v \in V} F_{\nu}(u, v)
$$


Let $\left\{U_{m}, m=1, \ldots\right\}$ be a decreasing sequence of neighborhoods of $x$ such that $\cap_{m \geqslant 1} U_{m}=\{x\}$. By the above to each such $U_{m}$ there corresponds $V_{m}$ such that for all $m$,

$$
\beta>\liminf _{\nu \rightarrow \infty} \sup _{v \in V_{m}} \inf _{u \in U_{m}} F_{\nu}(u, v) .
$$

Let $\left\{y_{\nu}, \nu=1, \ldots\right\}$ be a sequence $\sigma$-converging to $y$, then for each $\nu$ sufficiently large, $y_{\nu} \in V_{m}$ and thus

$$
\beta>\liminf _{\nu \rightarrow \infty} \inf _{u \in U_{m}} F_{\nu}\left(u, y_{\nu}\right) .
$$

The above and Corollary A.8 imply the existence of $\left\{u_{\nu, m}, \nu=1, \ldots, m=1, \ldots\right\}$ such that, for all $m, u_{\nu, m} \in U_{m}$ and

$$
\beta>\liminf _{\nu \rightarrow \infty} F_{\nu}\left(u_{\nu, m}, y_{\nu}\right)
$$

Thus

$$
\beta \geqslant \limsup _{m \rightarrow \infty} \liminf _{\nu \rightarrow \infty} F_{\nu}\left(u_{\nu, m}, y_{\nu}\right) .
$$

We now appeal to the standard diagonalization procedure to exhibit a sequence $\left\{x_{m}=u_{\nu_{m}, m}, m=1, \ldots\right\}$ such that

$$
\beta \geqslant \limsup _{m \rightarrow \infty} F_{\nu_{m}}\left(x_{m}, y_{\nu_{m}}\right)
$$

and hence

$$
\begin{aligned}
\beta & \geqslant \sup _{y_{\nu} \rightarrow \sigma y} \inf _{\substack{\left\{\nu_{n}\right\} \subset N \\
x_{m} \rightarrow \tau}} \liminf _{m \rightarrow \infty} F_{\nu_{m}}\left(x_{m}, y_{\nu_{m}}\right) \\
& =\sup _{y_{\nu} \rightarrow \sigma y} \inf _{x_{\nu} \rightarrow \tau} \liminf _{\nu \rightarrow \infty} F_{\nu}\left(x_{\nu}, y_{\nu}\right)=g(x, y) .
\end{aligned}
$$

From this the desired inequality follows since the above holds for every $\beta>l_{6} F_{\nu}$.

4.39 Corollary. Suppose $(X, \tau)$ and $(Y, \sigma)$ are metrizable and $\left\{F_{\nu}, \nu=1, \ldots\right\}$ is a sequence of saddle functions defined on $X \times Y$ with values in $\bar{R}$. Then the sequence $\left\{F_{\nu}\right.$, $\nu=1, \ldots\}$ converges to $F$ in the sense of $(4.31)$, i.e. $l_{1} F_{\nu} \leqslant F \leqslant l_{6} F_{\nu}$, or equivalently $l_{1} F_{\nu}=F=l_{6} F_{\nu}$ if and only if to any pair $(x, y)$

(i) there corresponds a sequence $\left\{y_{\nu}, \nu=1, \ldots\right\}$-convergent to $y$ such that for all sequences $\left\{x_{\nu}, \nu=1, \ldots\right\}$-convergent to $x$ we have

$$
F(x, y) \leqslant \liminf _{\nu \rightarrow \infty} F_{\nu}\left(x_{\nu}, y_{\nu}\right)
$$

and

(ii) there corresponds a sequence $\left\{x_{\nu}, \nu=1, \ldots\right\}$-convergent to $x$ such that for all sequences $\left\{y_{\nu}, \nu=1, \ldots\right\}$ o-convergent to $y$, we have

$$
F(x, y) \geqslant \limsup _{\nu \rightarrow \infty} F_{\nu}\left(x_{\nu}, y_{\nu}\right)
$$

We note that the sufficient conditions for epi/hypo-convergence given in Proposition 3.8 imply an even stronger type of convergence than $l_{1} F_{\nu}=F=l_{6} F_{\nu}$. In 
Corollary 4.39(i) the choice of $\left\{y_{\nu}, \nu=1, \ldots\right\}$ is allowed to depend on $x$ and $y$ not just on $y$ as in Proposition 3.8, and similarly the chioce of $\left\{x_{\nu}, \nu=1, \ldots\right\}$ depends on $x$ and $y$ in Corollary 4.39(ii) not just on $x$ as in Proposition 3.8. Thus, in addition to $l_{1} F_{\nu}=F=l_{6} F_{\nu}$, the convergence of saddle functions suggested by the conditions of Proposition 3.8 demands a uniformity property.

In [4] Cavazzuit calls a sequence of saddle functions $\left\{F_{\nu}: X \times Y \rightarrow \bar{R}, \nu=1, \ldots\right\}$ convergent to $F$ if $l_{e} F_{\nu}=F=l_{6} F_{\nu}$ which in view of the diagram of Figure 1 naturally implies that the sequence epi/hypo-converges to $F$ (and thus yields the convergence of the saddle points,...). Note that this definition demands that the limit function $F$ be $\tau$-lower semicontinuous in $x$ but there is no corresponding condition with respect to $y$.

Of special interest are the limit functions associated to a sequence whose elements are all the same saddle function $F$. We already know that if $\left\{F=F_{v}, \nu=1, \ldots\right\}$ then $l_{e} F_{\nu}$ and $l_{h} F_{\nu}$ may differ from $F$. cf. Example 4.8. However not all limit functions associated to such a sequence can be different, as can easily be assessed from their definitions. In such a situation we have

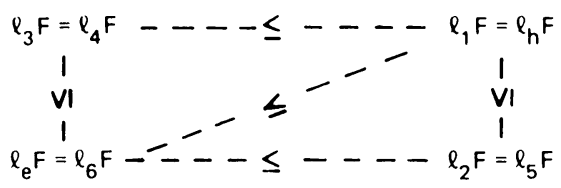

FIgURE 2. Limits of a single function

where we have written $l_{j} F$ rather than $l_{j} F_{\nu}$ to stress the fact that we are dealing with sequences whose elements are all identical to $F$, and again $\cdots \leqslant \cdots$ indicates the order relationship.

First noie that the sequence $\left\{F=F_{\nu}, \nu=1, \ldots\right\}$ always has an epi/hypo-limit $\left(l_{e} F \leqslant l_{h} F\right)$, but not a limit in the stronger sense of Corollary 4.39, cf. again Example 4.8. From the definition of $l_{3} F$ (or $l_{4} F$ ) and (2.1) and (2.18), it follows immediately that $l_{3} F=\mathrm{cl}_{y} \mathrm{cl}_{x} F=l_{4} F$ where $\mathrm{cl}_{y} G$ indicates that we take the upper closure of the function $y \mapsto G(x, y)$, for all $x \in X$ and $\operatorname{cl}_{x} G$ is the lower closure of the function $x \mapsto G(x, y)$ for all $y \in Y$, cf. §3. Similarly we get that $l_{2} F=\mathrm{cl}_{x} \mathrm{cl}_{y} F$ $=l_{5} F$. Note that the four limit functions could be different. In particular, we note that $l_{e} F \neq \mathrm{cl}_{x} \mathrm{cl}_{y} F$, and similarly $\mathrm{cl}_{y} \mathrm{cl}_{x} F \neq l_{h} F$.

4.40 EXAMPLE. Let

$$
F(x, y)= \begin{cases}0 & \text { if } x+y \text { is rational } \\ 1 & \text { if } x+y \text { is irrational. }\end{cases}
$$

Then $l_{e} F \equiv 0<1 \equiv \mathrm{cl}_{x} \mathrm{cl}_{y} F$.

We have seen that it is possible to associate to a sequence (or filtered collection) of saddle functions, various limit functions, which in turn can be used to define various types of convergence. We conclude by introducing one more class of limit functions involving extended closure. We shall say that a filtered collection of extended-real 
valued saddle functions $\left\{F_{\nu}, \nu \in N\right\}$ defined on $(X, \tau) \times(Y, \sigma)$ epi/hypo-converge to $F$ in the extended sense if

$$
\underline{\mathrm{cl}}_{x} l_{e} F_{\nu} \leqslant F \leqslant \overline{\mathrm{cl}}_{y} l_{h} F_{\nu}
$$

where by $\mathrm{cl}_{x}$ we mean the extended lower closure (3.4) with respect to $x$ for all $y$, and by $\overline{\mathrm{cl}}_{y}$ the extended upper closure (3.5) with respect to $y$ for all $x$. The interval of epi/hypo-limits associated to a collection of functions is, in general, smaller, as that defined by (4.41). We have shied away from using (4.41) as the central definition of our presentation because the extended closure adds a global property that cannot be translated in local characterization such as (3.6) and (3.7). However if the saddle functions are convex-concave, then the extended closures are naturally generated by conjugacy operations, cf. [10], and duality considerations lead us to work with this extended notion of epi/hypo-convergence. This is developed further in [3].

5. Yosida approximates: Metrizable case. Epi/hypo-convergence exhibits strong stability properties under various perturbations. This is already the case for epi-convergence, for example see $[\mathbf{1}, \mathbf{1 3}]$. This section is devoted to a special class of perturbations that generate a whole class of well-behaved approximates, called Yosida approximates. This type of approximation had already proved its utility in the study of epi-convergence, as well as that of partial differential equations of the evolution type, see $[\mathbf{1}, \mathbf{6}]$. The approach to epi/hypo-convergence via Yosida approximates, provides us with a powerful analytical tool in that it yields a concrete approximation scheme which can be used to study not only the epi/hypo-convergence of saddle functions, but also the convergence of the (differential) operators associated with saddle functions such as maximal monotone operators which are not necessarily the subdifferentials of convex functionals. In [3] we give a number of results in that direction.

At this point it is important to stress that, notwithstanding our exclusive reliance on perturbations that lead to Yosida approximates, the essence of our results is not really dependent on this specific type of perturbational or approximates. There are large classes of "natural" perturbations with whom we could achieve the same objectives. Rather than the square of the distance we could use any locally Lipschitz function of the distance, or even functions that are not directly related to the distance function (in the framework of epi-convergence, details can be found in [13, $\S 6])$, the choice of the approximation-type being conditioned by the application. In the Hilbert case, i.e. when both $(X, \tau)$ and $(Y, \sigma)$ are Hilbert spaces, or more generally in the Banach reflexive case, the use of the square of the distance is covenient, since epi/hypo-convergence properties are then intimately related to the convergence of the resolvents, cf. [1].

We take $(X, \tau)$ and $(Y, \sigma)$ to be metrizable and $d_{\tau}$ and $d_{\sigma}$ any metrics compatible with the topologies $\tau$ and $\sigma$ respectively. Let $F$ be a saddle function defined on $X \times Y$ with values in $\bar{R}$. For every $\lambda \geqslant 0, \mu>0$, we define

$$
F^{\downarrow}(\lambda, \mu, x, y)=\sup _{v \in Y} \inf _{u \in X}\left[F(u, v)+\frac{1}{2 \lambda} d_{\tau}^{2}(u, x)-\frac{1}{2 \mu} d_{\sigma}^{2}(v, y)\right]
$$


the lower Yosida approximate of $F$ (with parameters $\lambda, \mu$ ), and

$$
F^{\dagger}(\lambda, \mu, x, y)=\inf _{u \in X} \sup _{v \in Y}\left[F(u, v)+\frac{1}{2 \lambda} d_{\tau}^{2}(u, x)-\frac{1}{2 \mu} d_{\sigma}^{2}(v, y)\right]
$$

the upper Yosida approximate of $F$ (with parameters $\lambda, \mu$ ). Our terminology is justified by the fact that the $F^{\downarrow}$ and $F^{\uparrow}$ will play the role-and possess the same properties as-the Yosida approximates used to study the epi-convergence of univariate (convex) functions. In fact, when $F$ depends only on $x$, and not on $y$, then $F^{\downarrow}(\lambda, \cdot)=F^{\dagger}(\lambda, \cdot)$ is precisely the Yosida approximate [1]. The arguments presented here show that convexity is not intrinsically required to obtain an epi/hypoconvergence theory for saddle functions via Yosida approximates. This observation also applies to the univariate case, and thus many of the results here generalize those known in the study of epi-convergence (for convex functions).

At this point, the reader should be warned to resist the temptation to carry over the analogy with the univariate case too far. A substantial number of the results, and all the significant ones, depend on having $\lambda$ and $\mu$ play an independent role. Most of our analysis would fail if we had insisted on only a 1-parameter approximation family. Moreover, the fact that pointwise convergence of the Yosida approximates is equivalent to epi-convergence (of the Mosco-type, cf. [1, Theorem 1.2.d]), does not carry over to the bivariate case, not even when the functions are convex-concave and $X$ and $Y$ are finite dimensional. In fact, this would be somewhat unexpected because the definition of the epi/hypo-limit is in terms of two inequalities involving noncomparable functions! Finally note that all the subsequent results are metric invariant, i.e. the assertions remain valid whatever be the metrics $d_{\tau}$ and $d_{\sigma}$ used in the construction of $F^{\downarrow}$ and $F^{\uparrow}$. The actual Yosida approximates obviously depend on $d_{\tau}$ and $d_{\sigma}$, but they can be chosen so as to fit the application.

After some preliminaries, we show that the Yosida approximates are locally Lipschitz provided that the original functions are majorized and minorized in a sense to be made precise later on. The Lipschitz constants are explicitly computed. This allows us to assert later the locally equi-Lipschitz property of a collection of functions majorized and minorized by the same functions. This is a key ingredient in the proof of the main theorem of this section, which exhibits the relationship between epi/hypo-convergence and the convergence of the Yosida approximates.

Some of the results that follow depend on boundedness properties of the saddle functions. All conditions introduced here below presuppose the existence of some pair $u_{0} \in X$ and $v_{0} \in Y$ and a constant $r>0$. We say that $F$ is (quadratically) majorized on a slice (at $u^{0}$ ) if for all $v \in Y$,

$$
F\left(u_{0}, v\right) \leqslant r\left[d_{\sigma}^{2}\left(v, v_{0}\right)+1\right]
$$

it is minorized on a slice (at $v_{0}$ ) if for every $u \in X$,

$$
F\left(u, v_{0}\right) \geqslant-r\left[d_{\tau}^{2}\left(u, u_{0}\right)+1\right] .
$$

$F$ is said to be (quadratically) majorized if for all $u \in X$ and $v \in Y$,

$$
F(u, v) \leqslant r\left[d_{\tau}^{2}\left(u, u_{0}\right)+d_{\sigma}^{2}\left(v, v_{0}\right)+1\right],
$$


and finally minorized if for all $u \in X$ and $v \in Y$,

$$
F(u, v) \geqslant-r\left[d_{\tau}^{2}\left(u, u_{0}\right)+d_{\sigma}^{2}\left(v, v_{0}\right)+1\right] .
$$

In the statements of the results, we shall refer to both $F^{\downarrow}$ and $F^{\uparrow}$. It will always be sufficient to give the proof for either $F^{\downarrow}$ or $F^{\uparrow}$, since

$$
-F^{\dagger}(\lambda, \mu, x, y)=\sup _{x \in X} \inf _{v \in Y}\left[-F(u, v)+\frac{1}{2 \mu} d_{\sigma}^{2}(v, y)-\frac{1}{2 \lambda} d_{\tau}^{2}(u, x)\right]
$$

and thus $F^{\dagger}$ is the negative of the lower Yosida approximate (with parameters $\mu, \lambda$ ) of the saddle function $(-F)$ defined on $Y \times X$.

5.8 THEOREM. Suppose $\left(X, d_{\tau}\right)$ and $\left(Y, d_{\sigma}\right)$ are metric spaces and $F: X \times Y \rightarrow \bar{R}$ is a saddle function minorized on a slice (5.4) and majorized on a slice (5.3). Provided that $\lambda$ and $\mu$ are sufficiently small, the functions $y \mapsto F^{\downarrow}(\lambda, \mu, x, y)$ and $x \mapsto F^{\downarrow}(\lambda, \mu, x, y)$ are locally Lipschitz. Moreover, if, in addition, $F$ is minorized, then $F^{\downarrow}$ is locally Lipschitz in $(x, y)$ and if $F$ is majorized so is $F^{\uparrow}$.

Proof. In view of (5.7), it suffices to prove the theorem for $F^{\downarrow}$. We start by showing that $F^{\downarrow}$ is finite valued. From the definition (5.1) of $F^{\downarrow}$, it follows that

$$
F^{\downarrow}(\lambda, \mu, x, y) \leqslant \sup _{v \in Y}\left[F\left(u_{0}, v\right)+\frac{1}{2 \lambda} d_{\tau}^{2}\left(u_{0}, x\right)-\frac{1}{2 \mu} d_{\sigma}^{2}(v, y)\right] .
$$

Since $F$ is majorized on a slice (5.3), we have

$$
F^{\downarrow}(\lambda, \mu, x, y) \leqslant \frac{1}{2 \lambda} d_{\tau}^{2}\left(u_{0}, x\right)+\sup _{v \in Y}\left[r d_{\sigma}^{2}\left(v, v_{0}\right)+r-\frac{1}{2 \mu} d_{\sigma}^{2}(v, y)\right],
$$

which combined with the inequality

$$
d_{\sigma}^{2}\left(v, v_{0}\right) \leqslant 2 d_{\sigma}^{2}(v, y)+2 d_{\sigma}^{2}\left(y, v_{0}\right),
$$

implies that when $\mu \leqslant 1 / 4 r$,

$$
F^{\downarrow}(\lambda, \mu, x, y) \leqslant \frac{1}{2 \lambda} d_{\tau}^{2}\left(u_{0}, x\right)+r+2 r d_{\sigma}^{2}\left(y, v_{0}\right) .
$$

Again from the definition of $F^{\downarrow}$, it follows that

$$
F^{\downarrow}(\lambda, \mu, x, y) \geqslant \inf _{u \in X}\left[F\left(u, v_{0}\right)+\frac{1}{2 \lambda} d_{\tau}^{2}(u, x)-\frac{1}{2 \mu} d_{\sigma}^{2}\left(v_{0}, y\right)\right]
$$

which with (5.4) - recall that $F$ is minorized on a slice-yields

$$
F^{\downarrow}(\lambda, \mu, x, y) \geqslant-\frac{1}{2 \mu} d_{\sigma}^{2}\left(v_{0}, y\right)+\inf _{u \in X}\left[\frac{1}{2 \lambda} d_{\tau}^{2}(u, x)-r d_{\tau}^{2}\left(u, u_{0}\right)-r\right]
$$

and thus when $\lambda \leqslant 1 / 4 r$ we have that

$$
F^{\downarrow}(\lambda, \mu, x, y) \geqslant-\frac{1}{2 \mu} d_{\sigma}^{2}\left(v_{0}, y\right)-2 r d_{\tau}^{2}\left(u_{0}, x\right)-r .
$$

Combining (5.9) and (5.10), we see that when $0<\lambda \leqslant 1 / 4 r$ and $0<\mu \leqslant 1 / 4 r$, the functions $F^{\downarrow}(\lambda, \mu, \ldots)$ are finite valued, with bounds that depend continuously on the given parameters $u_{0}, v_{0}$ and $r$. 
For the remainder of the proof we may as well assume that $\lambda$ and $\mu$ are such that $F^{\downarrow}(\lambda, \mu, \cdot)$ is finite valued. For any pair $\varepsilon>0$ and fixed $y \in Y$, we can find $v^{\varepsilon}=v^{\varepsilon}(\lambda, \mu, x)$ such that

$$
\begin{aligned}
F^{\downarrow}(\lambda, \mu, x, y) & \geqslant \inf _{u \in X}\left[F\left(u, v^{\varepsilon}\right)+\frac{1}{2 \lambda} d_{\tau}^{2}(u, x)\right]-\frac{1}{2 \mu} d_{\sigma}^{2}\left(v^{\varepsilon}, y\right) \\
& \geqslant F^{\downarrow}(\lambda, \mu, x, y)-\varepsilon .
\end{aligned}
$$

In particular, this holds for $y=y_{1}$ and $y=y_{2}$ and $v^{\varepsilon}=v_{1}^{\varepsilon}$ and $v=v_{2}^{\varepsilon}$ respectively. We again use the definition of $F^{\downarrow}$ to obtain

$$
\begin{aligned}
F^{\downarrow}\left(\lambda, \mu, x, y_{1}\right) \geqslant & \inf _{u \in X}\left[F\left(u, v_{2}^{\varepsilon}\right)+\frac{1}{2 \lambda} d_{\tau}^{2}(u, x)\right]-\frac{1}{2 \mu} d_{\sigma}^{2}\left(v_{2}^{\varepsilon}, y_{1}\right) \\
& +\left[\frac{1}{2 \mu} d_{\sigma}^{2}\left(v_{2}^{\varepsilon}, y_{2}\right)-\frac{1}{2 \mu} d_{\sigma}^{2}\left(v_{2}^{\varepsilon}, y_{2}\right)\right] \\
\geqslant & F^{\downarrow}\left(\lambda, \mu, x, y_{2}\right)-\varepsilon+\frac{1}{2 \mu}\left[d_{\sigma}^{2}\left(v_{2}^{\varepsilon}, y_{2}\right)-d_{\sigma}^{2}\left(v_{2}^{\varepsilon}, y_{1}\right)\right]
\end{aligned}
$$

where the last relation follows from the second inequality in (5.11). Since $d_{\sigma}^{2}\left(v_{2}^{\varepsilon}, y_{1}\right)$ $-d_{\sigma}^{2}\left(v_{2}^{\varepsilon}, y_{2}\right) \leqslant d_{\sigma}\left(y_{1}, y_{2}\right)\left[2 d_{\sigma}\left(v_{2}^{\varepsilon}, y_{2}\right)+d_{\sigma}\left(y_{1}, y_{2}\right)\right]$, we have

$$
\begin{aligned}
& F^{\downarrow}\left(\lambda, \mu, x, y_{1}\right)-F^{\downarrow}\left(\lambda, \mu, x, y_{2}\right) \\
& \quad \geqslant-\varepsilon-\frac{1}{2 \mu} d_{\sigma}\left(y_{1}, y_{2}\right)\left[2 d_{\sigma}\left(v_{2}^{\varepsilon}, y_{2}\right)+d_{\sigma}\left(y_{1}, y_{2}\right)\right] .
\end{aligned}
$$

This, and a similar inequality obtained by the interchanging $y_{1}$ and $y_{2}$, yield

$$
\begin{aligned}
& \left|F^{\downarrow}\left(\lambda, \mu, x, y_{1}\right)-F^{\downarrow}\left(\lambda, \mu, x, y_{2}\right)\right| \\
& \quad \leqslant \varepsilon+\frac{1}{\mu} d_{\sigma}\left(y_{1}, y_{2}\right)\left[d_{0}\left(y_{1}, y_{2}\right)+d_{\sigma}\left(v_{1}^{\varepsilon}, y_{1}\right)+d_{\sigma}\left(v_{2}^{\varepsilon}, y_{2}\right)\right] .
\end{aligned}
$$

There remains only to obtain a local bound on $d_{\sigma}\left(v^{\varepsilon}, y\right)$. To do so we rely on the second inequality in (5.11), to get

$$
\inf _{u \in X}\left[F\left(u, v^{\varepsilon}\right)+\frac{1}{2 \lambda} d_{\tau}^{2}(u, x)\right]+\varepsilon \geqslant F^{\downarrow}(\lambda, \mu, x, y)+\frac{1}{2 \mu} d_{\sigma}^{2}\left(v^{\varepsilon}, y\right) .
$$

In particular with $u=u_{0}$ and $\lambda \leqslant 1 / 4 r$, from (5.10) we have

$$
\begin{aligned}
F\left(u_{0}, v^{\varepsilon}\right)+\frac{1}{2 \lambda} d_{\tau}^{2}\left(u_{0}, x\right) & +\varepsilon \\
\geqslant & -\frac{1}{2 \mu} d_{\sigma}^{2}\left(v_{0}, y\right)-2 r d_{\tau}^{2}\left(u_{0}, x\right)-r+\frac{1}{2 \mu} d_{\sigma}^{2}\left(v^{\varepsilon}, y\right) .
\end{aligned}
$$

Since $F$ is majorized on a slice, we use (5.3) to obtain

$$
\begin{aligned}
r\left[2 d_{\sigma}^{2}\left(v^{\varepsilon}, y\right)+2\right. & \left.d_{\sigma}^{2}\left(y, v_{0}\right)+1\right]+\frac{1}{2 \lambda} d_{\tau}^{2}\left(u_{0}, x\right)+\varepsilon \\
& +\frac{1}{2 \mu} d_{\sigma}^{2}\left(v_{0}, y\right)+r+2 r d_{\tau}^{2}\left(u_{0}, x\right) \geqslant \frac{1}{2 \mu} d_{\sigma}^{2}\left(v^{\varepsilon}, y\right) .
\end{aligned}
$$


If $\mu \leqslant 1 /(1+4 r)$, this yields

$$
d_{\sigma}^{2}\left(v^{\varepsilon}, y\right) \leqslant\left(4 r+\frac{1}{\mu}\right) d_{\sigma}^{2}\left(v_{0}, y\right)+\left(4 r+\frac{1}{\lambda}\right) d_{\tau}^{2}\left(u_{0}, x\right)+4 r+2 \varepsilon .
$$

Substituting in (5.12), we obtain

$$
\begin{aligned}
& \left|F^{\downarrow}\left(\lambda, \mu, x, y_{1}\right)-F^{\downarrow}\left(\lambda, \mu, x, y_{2}\right)\right| \\
& \qquad \varepsilon+d_{\sigma}\left(y_{1}, y_{2}\right) \cdot \frac{1}{\mu}\left\{d_{0}\left(y_{1}, y_{2}\right)+\left(4 r+\frac{1}{\mu}\right)\left[d_{\sigma}^{2}\left(v_{0}, y_{1}\right)+d_{\sigma}^{2}\left(v_{0}, y_{2}\right)\right]\right. \\
& \left.+2\left(4 r+\frac{1}{\lambda}\right) d_{\tau}^{2}\left(u_{0}, x\right)+8 r+4 \varepsilon\right\} .
\end{aligned}
$$

This holds for every $\varepsilon>0$, and thus we have that

$$
\begin{aligned}
& \left|F^{\downarrow}\left(\lambda, \mu, x, y_{1}\right)-F^{\downarrow}\left(\lambda, \mu, x, y_{2}\right)\right| \\
& \quad \leqslant d_{\sigma}\left(y_{1}, y_{2}\right) \cdot C_{2}\left(r, \mu, 1 / \mu, \lambda, 1 / \lambda, v_{0}, y_{1}, y_{2}\right)
\end{aligned}
$$

where $C_{2}$ is bounded on bounded subsets of its domain of definition. In particular for fixed $r, \mu, \lambda, u_{0}$ and $v_{0}, C_{2}$ is bounded above whenever $y_{1}$ and $y_{2}$ belong to some (fixed) bounded subset of $Y$. This yields the desired Lipschitz inequality.

A similar analysis, with $F^{\uparrow}$, rather than $F^{\downarrow}$, yields

$$
\begin{aligned}
& \left|F^{\dagger}\left(\lambda, \mu, x_{1}, y\right)-F^{\dagger}\left(\lambda, \mu, x_{2}, y\right)\right| \\
& \quad \leqslant d_{\tau}\left(x_{1}, x_{2}\right) \cdot C_{2}^{\prime}\left(r, \mu, 1 / \mu, \lambda, 1 / \lambda, v_{0}, x, y_{1}, y_{2}\right)
\end{aligned}
$$

the function $C_{2}^{\prime}$ being the same type of function as $C_{2}$.

Next we show that the function $x \mapsto F^{\downarrow}(\lambda, \mu, x, y)$ is locally Lipschitz if $F$ is also minorized (by a quadratic form), i.e. satisfies (5.6). For each $v \in Y$, we denote by

$$
[F(\cdot, v)]_{\lambda}(x)=\inf _{u \in X}\left[F(u, v)+\frac{1}{2 \lambda} d_{\tau}^{2}(u, x)\right]
$$

the (lower) Yosida approximate (with parameter $\lambda$ ) of the univariate function $u \mapsto F(u, v)$. With this notation, (5.11) becomes

$$
F^{\downarrow}(\lambda, \mu, x, y) \geqslant\left[F\left(\cdot, v^{\varepsilon}\right)\right]_{\lambda}(x)-\frac{1}{2 \mu} d_{\sigma}^{2}\left(v^{\varepsilon}, y\right) \geqslant F^{\downarrow}(\lambda, \mu, x, y)-\varepsilon .
$$

Recall that actually $v^{\varepsilon}$ depends not only on $\varepsilon$ but also on $\lambda, \mu, x$ and $y$. In the argument that follows, we shall keep $\lambda, \mu$ and $y$ fixed, but $x$ will vary. We now write

$$
v_{1}^{\varepsilon} \text { for } v\left(\varepsilon, \lambda, \mu, x_{1}, y\right), \quad v_{2}^{\varepsilon} \text { for } v\left(\varepsilon, \lambda, \mu, x_{2}, y\right) \text {, etc... }
$$

Again from the definition of $F^{\downarrow}$, we have that

$$
F^{\downarrow}\left(\lambda, \mu, x_{1}, y\right) \geqslant\left[F\left(\cdot, v_{2}^{\varepsilon}\right)\right]_{\lambda}\left(x_{1}\right)-\frac{1}{2 \mu} d_{\sigma}^{2}\left(v_{2}^{\varepsilon}, y\right)
$$

and hence

$$
\begin{aligned}
F^{\downarrow}\left(\lambda, \mu, x_{1}, y\right) \geqslant & {\left[F\left(\cdot, v_{2}^{\varepsilon}\right)\right]_{\lambda}\left(x_{2}\right)-\frac{1}{2 \mu} d_{\sigma}^{2}\left(v_{2}^{\varepsilon}, y\right) } \\
& +\left[F\left(\cdot, v_{2}^{\varepsilon}\right)\right]_{\lambda}\left(x_{1}\right)-\left[F\left(\cdot, v_{2}^{\varepsilon}\right)\right]_{\lambda}\left(x_{2}\right)
\end{aligned}
$$


which in view of $(5.17)$ yields

$$
\begin{aligned}
& F^{\downarrow}\left(\lambda, \mu, x_{2}, y\right)-F^{\downarrow}\left(\lambda, \mu, x_{1}, y\right) \\
& \quad \leqslant \varepsilon+\left[F\left(\cdot, v_{2}^{\varepsilon}\right)\right]_{\lambda}\left(x_{2}\right)-\left[F\left(\cdot, v_{2}^{\varepsilon}\right)\right]_{\lambda}\left(x_{1}\right) .
\end{aligned}
$$

Now from (5.16), the definition of $[F(\cdot, v)]_{\lambda}$, it follows that to each $\varepsilon>0, x \in X$, $v \in Y$ and $\lambda>0$, there corresponds a $u^{\varepsilon}=u^{\varepsilon}(\lambda, x, v)$, an $\varepsilon$-approximate infimum of the expression appearing in (5.16), such that

$$
\begin{aligned}
& {\left[F\left(\cdot, v_{2}^{\varepsilon}\right)\right]_{\lambda}\left(x_{2}\right)-\left[F\left(\cdot, v_{2}^{\varepsilon}\right)\right]_{\lambda}\left(x_{1}\right)} \\
& \quad \leqslant F\left(u_{1}^{\varepsilon}, v_{2}^{\varepsilon}\right)+\frac{1}{2 \lambda} d_{\tau}^{2}\left(u_{1}^{\varepsilon}, x_{2}\right)+\varepsilon-F\left(u_{1}^{\varepsilon}, v_{2}^{\varepsilon}\right)-\frac{1}{2 \lambda} d_{\tau}^{2}\left(u_{1}^{\varepsilon}, x_{1}\right)
\end{aligned}
$$

where

$$
\begin{aligned}
& u_{1}^{\varepsilon}=u^{\varepsilon}\left(\lambda, x_{1}, v_{2}^{\varepsilon}\right)=u^{\varepsilon}\left(\lambda, x_{1}, v\left(\varepsilon, \lambda, \mu, x_{2}, y\right)\right), \\
& u_{2}^{\varepsilon}=u^{\varepsilon}\left(\lambda, x_{2}, v_{2}^{\varepsilon}\right)=u^{\varepsilon}\left(\lambda, x_{2}, v\left(\varepsilon, \lambda, \mu, x_{2}, y\right)\right),
\end{aligned}
$$

and hence

$$
\begin{gathered}
{\left[F\left(\cdot, v_{2}^{\varepsilon}\right)\right]_{\lambda}\left(x_{2}\right)-\left[F\left(\cdot, v_{2}^{\varepsilon}\right)\right]_{\lambda}\left(x_{1}\right) \leqslant \frac{1}{2 \lambda}\left[d_{\tau}^{2}\left(u_{1}^{\varepsilon}, x_{2}\right)-d_{\tau}^{2}\left(u_{1}^{\varepsilon}, x_{1}\right)\right]+\varepsilon} \\
\leqslant \varepsilon+\frac{1}{2 \lambda} d_{\tau}\left(x_{1}, x_{2}\right)\left[2 d_{\tau}\left(u_{1}^{\varepsilon}, x_{1}\right)+d_{\tau}\left(x_{1}, x_{2}\right)\right] .
\end{gathered}
$$

We now seek an upper bound (or estimate) for $d_{\tau}\left(u_{1}^{\varepsilon}, x_{1}\right)$. From (5.3) and the definiton of $[F(\cdot, v)]_{\lambda}$ we also have that

$$
\begin{aligned}
{\left[F\left(\cdot, v_{2}^{\varepsilon}\right)\right]_{\lambda}\left(x_{1}\right) } & \leqslant F\left(u_{0}, v_{2}^{\varepsilon}\right)+\frac{1}{2 \lambda} d_{\tau}^{2}\left(u_{0}, x_{1}\right) \\
& \leqslant r\left[d_{\sigma}^{2}\left(v_{2}^{\varepsilon}, v_{0}\right)+1\right]+\frac{1}{2 \lambda} d_{\tau}^{2}\left(u_{0}, x_{1}\right) .
\end{aligned}
$$

We now use the fact that $F$ is minorized, i.e. condition (5.6) is satisfied, the definition of $u_{1}^{\varepsilon}$, and the previous inequality to conclude that

$$
\begin{aligned}
& 2 \varepsilon+r\left[d_{\sigma}^{2}\left(v_{2}^{\varepsilon}, v_{0}\right)+1\right]+\frac{1}{2 \lambda} d_{\tau}^{2}\left(u_{0}, x_{1}\right) \\
& \quad \geqslant-r\left[d_{\tau}^{2}\left(u_{1}^{\varepsilon}, u_{0}\right)+d_{\sigma}^{2}\left(v_{2}^{\varepsilon}, v_{0}\right)+1\right]+\frac{1}{2 \lambda} d_{\tau}^{2}\left(u_{1}^{\varepsilon}, x_{1}\right)
\end{aligned}
$$

This implies that if $\lambda \leqslant 1 / 2(1+2 r)$,

$$
d_{\tau}^{2}\left(u_{1}^{\varepsilon}, x_{1}\right) \leqslant 2 \varepsilon+2 r\left[d_{\sigma}^{2}\left(v_{2}^{\varepsilon}, v_{0}\right)+1\right]+\left(2+\frac{1}{2 \lambda}\right) d_{\tau}^{2}\left(u_{0}, x_{1}\right)
$$

which with (5.13) yields

$$
\begin{aligned}
d_{\tau}^{2}\left(u_{1}^{\varepsilon}, x_{1}\right) \leqslant & \varepsilon(2+8 r)+2 r(1+8 r)+4 r\left[1+\left(4 r+\frac{1}{\mu}\right)\right] d_{\sigma}^{2}\left(v_{0}, y\right) \\
& +\left(2 r+\frac{1}{2 \lambda}\right) d_{\tau}^{2}\left(u_{0}, x_{1}\right)+4 r\left(4 r+\frac{1}{\lambda}\right) d_{\tau}^{2}\left(u_{0}, x_{2}\right)
\end{aligned}
$$


Thus

$$
d_{\tau}\left(u_{1}^{\varepsilon}, x_{1}\right) \leqslant C_{1}\left(\varepsilon, r, \lambda, 1 / \lambda, \mu, 1 / \mu, u_{0}, v_{0}, x_{1}, x_{2}, y\right)
$$

where $C_{1}$ is bounded on a bounded region, in particular if $x_{1}$ and $x_{2}$ belong to a bounded subset of $X$ and $\varepsilon, r, \lambda$ and $\mu$ are fixed (with $\lambda$ and $\mu$ sufficently small).

Returning to (5.18), the second inequality of (5.20) and the inequality just derived for $d_{\tau}\left(u^{\varepsilon}\left(x_{1}, v^{\varepsilon}\left(x_{2}, y\right), x_{1}\right)\right)=d_{\tau}\left(u_{1}^{\varepsilon}, x_{1}\right)$, we get

$$
\begin{aligned}
& F^{\downarrow}\left(\lambda, \mu, x_{2}, y\right)-F^{\downarrow}\left(\lambda, \mu, x_{1}, y\right) \\
& \quad \leqslant 2 \varepsilon+\frac{1}{2 \lambda} d_{\tau}\left(x_{1}, x_{2}\right)\left[d_{\tau}\left(x_{1}, x_{2}\right)+2 C_{1}\left(\varepsilon, \lambda, \mu, x_{1}, x_{2}\right)\right] .
\end{aligned}
$$

Letting $\varepsilon$ go to 0 , for any bounded subset $S$ of $X$ we can find a constant $C_{1}$ (which depends only on $r, \lambda, \mu, u_{0}, v_{0}$ and $y$ ) such that for any $\left(x_{1}, x_{2}\right) \in S \times S$

$$
F^{\downarrow}\left(\lambda, \mu, x_{2}, y\right)-F^{\downarrow}\left(\lambda, \mu, x_{1}, y\right) \leqslant d_{\tau}\left(x_{1}, x_{2}\right) \cdot C_{1} .
$$

Reversing the role of $x_{1}$ and $x_{2}$, we have the (locally) Lipschitz inequality

$$
\left|F^{\downarrow}\left(\lambda, \mu, x_{1}, y\right)-F^{\downarrow}\left(\lambda, \mu, x_{2}, y\right)\right| \leqslant d_{\tau}\left(x_{1}, x_{2}\right) \cdot C_{1} \text {. }
$$

To show that $F^{\downarrow}(\lambda, \mu, \cdot)$ is locally Lipschitz in $(x, y)$ it now suffices to observe that from (5.14) and (5.22) it follows that

$$
\begin{aligned}
\mid F^{\downarrow}(\lambda, \mu, & \left.x_{1}, y_{1}\right)-F^{\downarrow}\left(\lambda, \mu, x_{2}, y_{2}\right) \mid \\
& \leqslant\left|F^{\downarrow}\left(\lambda, \mu, x_{1}, y_{1}\right)-F^{\downarrow}\left(\lambda, \mu, x_{2}, y_{1}\right)\right| \\
& +\left|F^{\downarrow}\left(\lambda, \mu, x_{2}, y_{1}\right)-F^{\downarrow}\left(\lambda, \mu, x_{2}, y_{2}\right)\right| \\
& \leqslant d_{\tau}\left(x_{1}, x_{2}\right) \cdot C_{1}+d_{\tau}\left(y_{1}, y_{2}\right) \cdot C_{2} \\
& \leqslant C\left(\lambda, \mu, x_{1}, y_{1}, x_{2}, y_{2}\right)\left[d_{\tau}\left(x_{1}, x_{2}\right)+d_{\sigma}\left(y_{1}, y_{2}\right)\right],
\end{aligned}
$$

and this completes the proof that $F^{\downarrow}$ is locally Lipschitz in $(x, y)$ if $F$ is majorized on a slice (5.3) and (totally) minorized (5.6).

As already indicated at the outset, the proof for $F^{\downarrow}$ proceeds along the same lines, except that this time we need $F$ to be minorized on a slice (5.4) and majorized (5.5).

The next theorem yields a complete characterization of the limit functions associated to a sequence $\left\{F_{\nu}, \nu=1, \ldots\right\}$ of saddle functions in terms of limits of the Yosida approximates of the $F_{\nu}$.

5.24 ThEOREM. Suppose $\left\{F_{\nu}, \nu=1, \ldots\right\}$ is a sequence of extended-real valued saddle functions defined on the product of the metric spaces $\left(X, d_{\tau}\right)$ and $\left(Y, d_{\sigma}\right)$. If the $F_{\nu}$ are uniformly majorized on a slice and minorized, i.e. the parameters $u_{0}, v_{0}$ and $r$ that appear in (5.3) and (5.6) are independent of $\nu$, then

$$
l_{e} F_{\nu}(x, y)=\sup _{\lambda>0} \inf _{\mu>0} \limsup _{\nu \rightarrow \infty} F_{\nu}^{\downarrow}(\lambda, \mu, x, y) .
$$

On the other hand, if the $F_{\nu}$ are uniformly minorized on a slice and majorized, then

$$
l_{h} F_{\nu}(x, y)=\inf _{\mu>0} \sup _{\lambda>0} \liminf _{\nu \rightarrow \infty} F_{\nu}^{\uparrow}(\lambda, \mu, x, y) .
$$


Thus, if the sequence $\left\{F_{\nu}, \nu=1, \ldots\right\}$ is uniformly minorized and majorized, it epi/hypo-converges to $F$ if and only if

(5.27) $\sup _{\lambda>0} \inf _{\mu>0} \limsup _{\nu \rightarrow \infty} F_{\nu}^{\downarrow}(\lambda, \mu, x, y) \leqslant F \leqslant \inf _{\mu>0} \sup _{\lambda>0} \liminf _{\nu \rightarrow \infty} F_{\nu}^{\uparrow}(\lambda, \mu, x, y)$.

Proof. In view of (5.7) it clearly suffices to prove any one of the identities (5.25) or (5.26). We choose to derive (5.26). We show first that

$$
a=\inf _{\mu>0} \sup _{\lambda>0} \liminf _{\nu \rightarrow \infty} F_{\nu}^{\uparrow}(\lambda, \mu, x, y) \geqslant l_{h} F_{\nu}(x, y) .
$$

Clearly there is nothing to prove if $a=+\infty$, thus suppose that $a<+\infty$. Take any $\beta>a$. We shall show that $\beta \geqslant l_{h} F_{\nu}(x, y)$. Since $\beta>a$, there exists $\mu_{0}>0$ such that

$$
\beta>\sup _{\lambda>0} \liminf _{\nu \rightarrow \infty} F_{\nu}^{\dagger}\left(\lambda, \mu_{0}, x, y\right) \text {. }
$$

Since $F_{\nu}^{\uparrow}$ is finite valued (cf. Theorem 5.8), from (5.2), the definition of $F_{\nu}^{\uparrow}$, it follows that for any $\lambda>0$ and $\nu$, there exists $u_{\nu}^{\lambda}$ such that

$$
F_{\nu}^{\dagger}\left(\lambda, \mu_{0}, x, y\right) \geqslant-\nu^{-1}+\sup _{v \in Y}\left[F_{\nu}\left(u_{\nu}^{\lambda}, v\right)-\frac{1}{2 \mu_{0}} d_{\sigma}^{2}(v, y)\right]+\frac{1}{2 \lambda} d_{\tau}^{2}\left(u_{\nu}^{\lambda}, x\right) .
$$

Hence

$$
\beta>\liminf _{\lambda \downarrow 0} \liminf _{\nu \rightarrow \infty}\left(\sup _{v \in Y}\left[F_{\nu}\left(u_{\nu}^{\lambda}, v\right)-\frac{1}{2 \mu_{0}} d_{\sigma}^{2}(v, y)\right]+\frac{1}{2 \lambda} d_{\tau}^{2}\left(u_{\nu}^{\lambda}, x\right)\right) .
$$

Lemma A.1, proved in the Appendix, allows us to diagonalize this double indexed sequence so that

$$
\beta>\liminf _{\nu \rightarrow \infty}\left\{\sup _{v \in Y}\left[F_{\nu}\left(x_{\nu}, v\right)-\frac{1}{2 \mu_{0}} d_{\sigma}^{2}(v, y)\right]+\frac{1}{2 \lambda(\nu)} d_{\tau}^{2}\left(x_{\nu}, x\right)\right\}
$$

for some choice of $\{\lambda(\nu), \nu=1, \ldots\}$ where $x_{\nu}=u_{\nu}^{\lambda(\nu)}$. By definition of lim inf, there exists a subsequence of indices such that

$$
\beta>\lim _{k \rightarrow \infty}\left(\sup _{v \in Y}\left[F_{\nu_{k}}\left(x_{k}, v\right)-\frac{1}{2 \mu_{0}} d_{\sigma}^{2}(v, y)\right]+\frac{1}{2 \lambda_{k}} d_{\tau}^{2}\left(x_{k}, x\right)\right),
$$

where for $k=1, \ldots, x_{k}=x_{\nu_{k}}$ and $\lambda_{k}=\lambda\left(\nu_{k}\right)$. Since we are taking a supremum with respect to $v$, taking $v=v_{0}$ and using (5.4), we get that

$$
\beta>\limsup _{k \rightarrow \infty}\left[-r\left(d_{\tau}^{2}\left(x_{k}, u_{0}\right)+1\right)-\frac{1}{2 \mu_{0}} d_{\sigma}^{2}\left(v_{0}, y\right)+\frac{1}{2 \lambda_{k}} d_{\tau}^{2}\left(x_{k}, x\right)\right] .
$$

Since $\lambda_{k}$ goes to 0 as $k$ goes to $\infty$, this implies that the $x_{k}$ converge to $x$. Because $d_{\tau}^{2}\left(x_{k}, x\right) \geqslant 0$. From (5.29) we obtain

$$
\beta>\limsup \sup _{k \rightarrow \infty}\left[F_{\nu_{k}}\left(x_{k}, v\right)-\frac{1}{2 \mu_{0}} d_{\sigma}^{2}(v, y)\right] .
$$

Thus for any sequence $y_{k}$ converging to $y$ we have that

$$
\beta>\limsup _{k \rightarrow \infty}\left[F_{\nu_{k}}\left(x_{k}, y_{k}\right)-\frac{1}{2 \mu_{0}} d_{\sigma}^{2}\left(y_{k}, y\right)\right],
$$


which implies

$$
\beta \geqslant \sup _{y_{k} \rightarrow y} \limsup _{k \rightarrow \infty}\left[F_{\nu_{k}}\left(x_{k}, y_{k}\right)\right]
$$

and this in turn yields

$$
\beta \geqslant \inf _{\substack{\left\{\nu_{k}\right\} \\ x_{k} \rightarrow x}} \sup _{y_{k} \rightarrow y} \limsup _{k \rightarrow \infty} F_{\nu_{k}}\left(x_{k}, y_{k}\right)=l_{h} F_{\nu}(x, y),
$$

and hence completes the proof of (5.28).

Next we derive the converse inequality, namely that

$$
a=\inf _{\mu>0} \sup _{\lambda>0} \liminf _{\nu \rightarrow \infty} F_{\nu}^{\dagger}(\lambda, \mu, x, y) \leqslant l_{h} F_{\nu}(x, y) .
$$

If $a=-\infty$, the inequality is trivially satisfied. Thus let us assume that $a>-\infty$. From the sequential definition of $l_{h}$, cf. Theorem 4.10, to prove (5.30), we need to show that for every subsequence of functions $\left\{F_{\nu_{k}}, k=1, \ldots\right\}$ and every sequence $\left\{x_{k}, k=1, \ldots\right\}$ converging to $x$, there exist $\left\{y_{k}, k=1, \ldots\right\}$ converging to $y$ such that

$$
a \leqslant \liminf _{k \rightarrow \infty} F_{k}\left(x_{k}, y_{k}\right)
$$

where we write simply $F_{k}$ for $F_{\nu_{k}}$.

The hypotheses of the theorem imply that the upper Yosida approximates $F_{\nu}^{\uparrow}$ are locally equi-Lipschitz with respect to $x$, as follows from Theorem 5.8 since we can use the same Lipschitz constants (5.23). Making use of this fact, to obtain the first inequality below, we get

$$
\begin{aligned}
\liminf _{\nu \rightarrow \infty} F_{\nu}^{\uparrow} & (\lambda, \mu, x, y) \leqslant \liminf _{k \rightarrow \infty} F_{k}^{\uparrow}\left(\lambda, \mu, x_{k}, y\right) \\
& =\liminf _{k \rightarrow \infty} \sup _{u \in X} \sup _{v \in Y}\left[F_{k}(u, v)+\frac{1}{2 \lambda} d_{\tau}^{2}\left(u, x_{k}\right)-\frac{1}{2 \mu} d_{\sigma}^{2}(v, y)\right] \\
& \leqslant \liminf _{k \rightarrow \infty} \sup _{v \in Y}\left[F_{k}\left(x_{k}, v\right)-\frac{1}{2 \mu} d_{\sigma}^{2}(v, y)\right] \\
& \leqslant \liminf _{k \rightarrow \infty}\left[F_{k}\left(x_{k}, v_{k}^{\mu}\right)-\frac{1}{2 \mu} d_{\sigma}^{2}\left(v_{k}^{\mu}, y\right)\right]
\end{aligned}
$$

where for every $k$ and $\mu>0, v_{k}^{\mu}$ is given by Lemma A.5.

This last bound for $\liminf \operatorname{in}_{\nu \rightarrow \infty} F_{\nu}^{\uparrow}$ being independent of $\lambda$, we get

$$
\sup _{\lambda>0} \liminf _{\nu \rightarrow \infty} F_{\nu}^{\uparrow}(\lambda, \mu, x, y) \leqslant \liminf _{k \rightarrow \infty}\left[F_{k}\left(x_{k}, v_{k}^{\mu}\right)-\frac{1}{2 \mu} d_{\sigma}^{2}\left(v_{k}^{\mu}, y\right)\right] .
$$

Taking the infimum with respect to $\mu$, or equivalently the limit when $\mu$ goes to 0 - see the definition of $F^{\downarrow}$ -

$$
\begin{aligned}
a & =\inf _{\mu>0} \sup _{\lambda>0} \liminf _{\nu \rightarrow \infty} F_{\nu}^{\uparrow}(\lambda, \mu, x, y) \\
& \leqslant \liminf _{\mu \rightarrow 0} \liminf _{k \rightarrow \infty}\left[F_{k}\left(x_{k}, v_{k}^{\mu}\right)-\frac{1}{2 \mu} d_{\sigma}^{2}\left(v_{k}^{\mu}, y\right)\right] .
\end{aligned}
$$


To diagonalize this sequence double indexed by $\mu$ and $k$, we apply the diagonalization Lemma A.1 which yields a subsequence of indices $\{\mu(k), k=1, \ldots\}$ decreasing with $k$ to 0 , such that

$$
a \leqslant \liminf _{k \rightarrow \infty}\left[F_{k}\left(x_{k}, y_{k}\right)-\frac{1}{2 \mu_{k}} d_{o}^{2}\left(y_{k}, y\right)\right]
$$

where for $k=1, \ldots, y_{k}=v_{k}^{\mu(k)}$ and $\mu_{k}=\mu(k)$; thus $\mathrm{a} \leqslant \liminf _{k \rightarrow \infty} F_{k}\left(x_{k}, y_{k}\right)$. There remains only to show that the $y_{k}$ converge to $y$. Since $a>-\infty$, (5.32) implies that for $k$ sufficiently large

$$
\frac{1}{2 \mu_{k}} d_{\sigma}^{2}\left(y_{k}, y\right) \leqslant F_{k}\left(x_{k}, y_{k}\right)+1-a .
$$

We now use (5.5) to obtain an upper bound for $d_{\sigma}^{2}\left(y_{k}, y\right)$, namely

$$
\frac{1}{2 \mu_{k}} d_{\sigma}^{2}\left(y_{k}, y\right) \leqslant r\left[d_{\tau}^{2}\left(x_{k}, u_{0}\right)+d_{\sigma}^{2}\left(y_{k}, v_{0}\right)+1\right]+1-a
$$

which implies that the $y_{k}$ converge to $y$ (recall that the $\mu_{k}$ tend to 0 ).

The next corollary justifies the use of the terminology "Yosida approximates" when referring to the function $F^{\downarrow}(\lambda, \mu, \ldots)$ and $F^{\dagger}(\lambda, \mu, \ldots)$.

5.33 Corollary. Suppose $F$ is a saddle function defined on the product of the metric spaces $\left(X, d_{\tau}\right)$ and $\left(Y, d_{\sigma}\right)$ with values in the extended reals. Suppose $F$ is majorized on a slice (5.3) and minorized (5.6). Then

$$
l_{e} F(x, y)=\sup _{\lambda>0} \inf _{\mu>0} F^{\downarrow}(\lambda, \mu, x, y) .
$$

Similarly, if $F$ is minorized on a slice (5.4) and majorized (5.5), then

$$
l_{h} F(x, y)=\inf _{\mu>0} \sup _{\lambda>0} F^{\dagger}(\lambda, \mu, x, y) .
$$

Finally if $F$ is both (quadratically) majorized and minorized, then

$$
l_{e} F=\sup _{\lambda>0} \inf _{\mu>0} F^{\downarrow}(\lambda, \mu, \ldots) \leqslant \inf _{\lambda>0} \sup _{\mu>0} F^{\dagger}(\lambda, \mu, \ldots)=l_{h} F .
$$

We terminate with a comparison of Theorem 5.24 and those relating the convergence of the Yosida approximates and the epi-convergence of convex functionals, in particular Theorem 1.2 of [1]. To do this it is useful to restrict oneself to the epi-convergence (univariate) version of Theorem 5.24.

5.37 THEOREM. Suppose that $\left\{f_{\nu}, \nu=1, \ldots\right\}$ is a sequence of extended-real valued functions defined on the metric space $\left(X, d_{\tau}\right)$. Suppose, moreover, that there exists $x_{0} \in X$ and $r>0$ such that for $\nu=1, \ldots$,

$$
f_{\nu}(x) \geqslant-r\left[d_{\tau}^{2}\left(x, x_{0}\right)+1\right]
$$

Then $f=\lim _{e} f$ if and only if

$$
f=\sup _{\lambda>0} \limsup _{\nu \rightarrow \infty}\left[f_{\nu}\right]_{\lambda}=\sup _{\lambda>0} \liminf _{\nu \rightarrow \infty}\left[f_{\nu}\right]_{\lambda}
$$

where $\left[f_{\nu}\right]_{\lambda}$ is the Yosida approximate with parameter $\lambda$ as defined by (2.17). 
The reader should note that even in finite dimensions, contrary to the "convex case", cf. Theorem $1.2 \mathrm{~d}$ of [1], we cannot assert the equivalence between epiconvergence and the pointwise convergence of the Yosida approximates to the Yosida approximate of $f$ (for fixed $\lambda$ ). This parallels the situation when characterizing the convergence of closed convex sets and that of just closed sets through $r$-convergence, cf. [11, part (vii) of the theorem and the remarks on p. 156].

6. A compactness theorem. The purpose of this section is to show that when $(X, \tau)$ and $(Y, \sigma)$ are metrizable and separable, every sequence of saddle functions contains an epi/hypo-convergent subsequence. This generalizes (and contains) Theorem 2.6, a similar result for univariate functions and epi-convergence. The proof is completely novel. We rely on the properties of the Yosida approximates and the characterization of epi/hypo-convergence in terms of the convergence of the Yosida approximates. When specialized to the case of univariate functions, we also obtain a new proof of Theorem 2.6. (The proof found in Kuratowski's book [8, p. 246] is essentially the standard argument used to porve the Bolzano-Weierstrass Theorem adapted to fit sequences of sets.) Finally, let us note that this approach sheds some light on the limiting process and to some extent it allows us to identify the limit element of the convergent subsequence.

6.1 TheOREM. Suppose $(X, \tau)$ and $(Y, \sigma)$ are both separable and metrizable. Then any sequence of saddle functions $\left\{F_{\nu}: X \times Y \rightarrow \bar{R}, \nu=1, \ldots\right\}$ contains a subsequence which is $\tau$-epi/o-hypo-convergent.

The proof is in three parts. We first demonstrate that the theorem holds when the saddle functions are bounded; actually all that is necessary for this first step is that they be majorized (5.5) and minorized (5.6). The next step is to prove the theorem when the saddle functions are just bounded below. Finally the last step yields the proof of the theorem in its full generality.

Step 1. We prove the theorem under the additional assumption that the $\left\{F_{\nu}\right.$, $\nu=1, \ldots\}$ are uniformly bounded on $X \times Y$. From Theorem 5.8, in particular (5.14), (5.22) and (5.23), lower and upper Yosida approximates $F_{\nu}{ }^{\downarrow}(\lambda, \mu, \ldots)$ and $F_{\nu}^{\dagger}(\lambda, \mu, \ldots)$ are locally equi-Lipschitz (in $x$ and $y$ ). Take $\left\{x_{p}, p=1, \ldots\right\}$ a countable dense subset of $X$ and $\left\{y_{q}, q=1, \ldots\right\}$ a countable dense subset of $Y$. Let $\left\{\lambda_{n}\right.$, $n=1, \ldots\}$ and $\left\{\mu_{m}, m=1, \ldots\right\}$ be two decreasing sequences that tend to 0 . For each $(n, m, p, q)$ the sequences $\left\{F_{\nu}^{\uparrow}\left(\lambda_{n}, \mu_{m}, x_{p}, y_{q}\right), \nu=1, \ldots\right\}$ and $\left\{F_{\nu}{ }^{\downarrow}\left(\lambda_{n}, \mu_{m}, x_{p}, y_{q}\right), \nu=1, \ldots\right\}$ are bounded in $R$ and thus relatively compact. By a standard diagonalization argument we can extract a subsequence $\nu_{k}$ such that for all $n, m, p, q$ the limit of the sequences $\left\{F_{\nu_{k}}^{\uparrow}\left(\lambda_{n}, \mu_{m}, x_{p}, y_{q}\right), k=1, \ldots\right\}$ and $\left\{F_{\nu_{k}}^{\downarrow}\left(\lambda_{n}, \mu_{m}, x_{p}, y_{q}\right), k=1, \ldots\right\}$ exist.

The equi-Lipschitz property implies that the sequences

$$
\left\{F_{\nu_{k}}^{\downarrow}\left(\lambda_{n}, \mu_{m}, x, y\right), k=1, \ldots\right\} \quad \text { and } \quad\left\{F_{\nu_{k}}^{\uparrow}\left(\lambda_{n}, \mu_{m}, x, y\right), k=1, \ldots\right\}
$$

converge for every $\left(\lambda_{n}, \mu_{m}, x, y\right)$ with $n=1, \ldots, m=1, \ldots, x \in X$ and $y \in Y$. 
From Theorem 5.24, we know that

$$
\begin{aligned}
l_{e} F_{\nu_{k}}(x, y) & =\sup _{\lambda>0} \inf _{\mu>0} \limsup _{k \rightarrow \infty} F_{\nu_{k}}^{\downarrow}(\lambda, \mu, x, y) \\
& =\sup _{\lambda_{n} \downarrow 0} \inf _{\mu_{m} \downarrow 0} \limsup _{k \rightarrow \infty} F_{\nu_{k}}^{\downarrow}\left(\lambda_{n}, \mu_{m}, x, y\right)
\end{aligned}
$$

where the second equality follows from the monotonicity of the functions $\lambda \mapsto$ $F_{\nu}{ }^{\downarrow}(\lambda, \mu, x, y)$ and $\mu \mapsto F_{\mu}^{\downarrow}(\lambda, \mu, x, y)$. Similarly, from Theorem 5.24, we have that

$$
\begin{aligned}
l_{h} F_{\nu_{k}}(x, y) & =\inf _{\mu>0} \sup _{\lambda>0} \liminf _{k \rightarrow \infty} F_{\nu_{k}}^{\dagger}(\lambda, \mu, x, y) \\
& =\inf _{\mu_{m} \downarrow 0} \sup _{\lambda_{n} \downarrow 0} \liminf _{k \rightarrow \infty} F_{\nu_{k}}^{\dagger}\left(\lambda_{n}, \mu_{m}, x, y\right),
\end{aligned}
$$

the second again following from the montonicity of $F^{\uparrow}$ in $\lambda$ and $\mu$.

Since $F^{\uparrow} \geqslant F^{\downarrow}$, since $\liminf _{k \rightarrow \infty} F_{\nu_{k}}^{\downarrow}$ and $\lim \sup _{k \rightarrow \infty} F_{\nu_{k}}^{\downarrow}$ are equal as follows from our choice of the sequence $\left\{\nu_{k}\right\}$, and since inf sup $\geqslant$ sup inf, we have that

$$
\begin{aligned}
l_{e} F_{\nu_{k}}(x, y) & =\sup _{\lambda_{n} \downarrow 0} \inf _{\mu_{m} \downarrow 0} \lim _{k \rightarrow \infty} F_{\nu_{k}}^{\downarrow}\left(\lambda_{n}, \mu_{m}, x, y\right) \\
& \leqslant \inf _{\mu_{m} \downarrow 0} \sup _{\lambda_{n} \downarrow 0} \lim _{k \rightarrow \infty} F_{\nu_{k}}^{\downarrow}\left(\lambda_{n}, \mu_{m}, x, y\right)=l_{h} F_{\nu_{k}}(x, y) .
\end{aligned}
$$

Thus, we have found a sequence $\left\{F_{\nu_{k}}, k=1, \ldots\right\}$ such that

$$
l_{e} F_{\nu_{k}} \leqslant l_{h} F_{\nu_{k}}
$$

which is precisely the definition of epi/hypo-convergence, see (4.5) and (4.6).

Step 2. We now assume that the saddle functions are bounded below, say by a constant $r$. For each $q=1, \ldots$ we define $F_{\nu}^{q}$, the truncation of $F_{\nu}$ from above, i.e. $F_{\nu}^{q}=\inf \left(F_{\nu}, q\right)$. For each $q$, the sequence of saddle functions $\left\{F_{\nu}^{q}, \nu=1, \ldots\right\}$ satisfies the hypotheses laid out in Step 1. Thus for each $q$, we can find a subsequence of saddle functions which is epi/hypo-convergent. We can now rely on the standard diagonalization procedure to extract a subsequence $\left\{F_{\nu_{k}}, k=1, \ldots\right\}$ such that the sequences $\left\{F_{\nu_{k}}^{q}, k=1, \ldots\right\}$ converge for each $q$. For $q=1, \ldots$, define

$$
F^{q}=\operatorname{lm}_{e / h} F_{\nu_{k}}^{q} \text {. }
$$

Note that the sequence $\left\{F^{q}, q=1, \ldots\right\}$ is montone increasing. Let $F=\sup _{q} F^{q}$ and we shall prove that $F=\operatorname{lm}_{e / h} F_{\nu_{k}}$, i.e. that $l_{e} F_{\nu_{k}} \leqslant F \leqslant l_{h} F_{\nu_{k}}$.

Since $F_{\nu_{k}}^{q} \leqslant F_{\nu_{k}}$, it follows that for all $q=1, \ldots, l_{h} F_{\nu_{k}}^{q} \leqslant l_{h} F_{\nu_{k}}$. By definition $F^{q}=\operatorname{lm}_{e / h} F_{\nu_{k}}^{q} \leqslant l_{h} F_{\nu_{k}}^{q}$ and thus for every $q, F^{q} \leqslant l_{h} F_{\nu_{k}}$ and consequently $F=$ $\sup F^{q} \leqslant l_{h} F_{\nu_{k}}$. It remains to show that $l_{e} F_{\nu_{k}} \leqslant F$. If $F(x, y)=+\infty$, the inequality is trivially satisfied. Henceforth suppose that $F(x, y)<+\infty$. We have to prove that given any subsequence of functions $\left\{F_{\nu_{p}}, p=1, \ldots\right\} \subset\left\{F_{\nu_{k}}, k=1, \ldots\right\}$, any sequence $\left\{y_{p}, p=1, \ldots\right\}$ converging to $y$, there exists a sequence $\left\{x_{p}, p=1, \ldots\right\}$ converging to $x$ such that

$$
F(x, y) \geqslant \underset{p \rightarrow \infty}{\limsup } F_{\nu_{p}}\left(x_{p}, y_{p}\right)
$$


Since for each $q, F^{q}=\operatorname{lm}_{e / h} F_{\nu_{p}}^{q} \geqslant l_{e} F_{\nu_{p}}^{q}$, there exists a sequence $\left\{x_{p}^{q}, p=1, \ldots\right\}$ converging to $x$ such that

$$
F^{q}(x, y) \geqslant \limsup _{p \rightarrow \infty} F_{\nu_{p}}^{q}\left(x_{p}^{q}, y_{p}\right) .
$$

Because $F(x, y)<\infty$ and, $F(x, y) \geqslant F^{q}(x, y) \geqslant \lim \sup _{p \rightarrow \infty} F_{\nu_{p}}^{q}\left(x_{p}^{q}, y_{p}\right)=$ $\lim \sup _{p \rightarrow \infty} \inf \left[F_{\nu_{p}}\left(x_{p}^{q}, y_{p}\right), q\right]$, for any $q>F(x, y)$, this last expression becomes

$$
F(x, y) \geqslant F^{q}(x, y) \geqslant \underset{p \rightarrow \infty}{\limsup } F_{\nu_{p}}\left(x_{p}^{q}, y_{p}\right),
$$

and thus by definition of $F$,

$$
F(x, y) \geqslant \underset{q \rightarrow \infty}{\limsup } \limsup _{p \rightarrow \infty} F_{\nu_{p}}\left(x_{p}^{q}, y_{p}\right) .
$$

Note that since for all $q$ the $\left\{x_{p}^{q}, p=1, \ldots\right\}$ converge to $x$, we also have

$$
0 \geqslant \limsup _{q \rightarrow \infty} \limsup _{p \rightarrow \infty} d\left(x_{p}^{q}, x\right) \text {. }
$$

The diagonalization Lemma A.3, actually an obvious but slightly modified version, allows us to extract a subsequence $\left\{x_{p}^{q(p)}=x_{p}, p=1, \ldots\right\}$ such that both

$$
F(x, y) \geqslant \limsup _{p \rightarrow \infty} F_{\nu_{p}}\left(x_{p}, y_{p}\right) \text { and } 0 \geqslant \limsup _{p \rightarrow \infty} d\left(x_{p}, x\right) .
$$

Thus we have exhibited the sought-for sequence converging to $x$ for which (6.2) is satisfied.

Step 3 . We are now ready to actually prove the assertion of the theorem without any restrictions on the $F_{\nu}$. For every. $q$, we define $F_{\nu}^{q}=\sup \left(F_{\nu},-q\right) \geqslant-q$. The sequence $\left\{F_{\nu}^{q}, \nu=1, \ldots\right\}$ is bounded from below and thus we are in the setting of Step 2. Hence for each $q$ we can find a sequence that epi/hypo-converges to a (limit) saddle function. The standard diagonalization argument allows us to extract a subsequence $\left\{F_{\nu_{k}}, k=1, \ldots\right\}$ such that for each $q$, the sequences $\left\{F_{\nu_{k}}^{q}, k=1, \ldots\right\}$ are epi/hypo-converging. For $q=1, \ldots$, define $F^{q}=\operatorname{lm}_{e / h} F_{\nu_{k}}^{q}$ The sequence $\left\{F^{q}\right.$, $q=1, \ldots\}$ is monotone decreasing and thus the saddle function $F=\inf _{q} F^{q}$, with values in $\bar{R}$ is well defined. As in Step 2, we shall prove that $F=\operatorname{lm}_{e / h} F_{\nu_{h}}$, i.e. that $l_{e} F_{\nu_{k}} \leqslant F \leqslant l_{h} F_{\nu_{k}}$.

Since $F_{\nu_{k}}^{q} \geqslant F_{\nu_{k}}$, it follows that for all $q=1, \ldots, l_{e} F_{\nu_{k}}^{q} \geqslant l_{e} F_{\nu_{k}}$. By definition $F^{q}=\operatorname{lm}_{e / h} F_{\nu_{k}}^{q} \geqslant l_{e} F_{\nu_{k}}^{q}$ and thus for all $q, F^{q} \geqslant l_{e} F_{\nu_{k}}$. Consequently $F=\inf _{q} F^{q} \geqslant$ $l_{e} F_{\nu_{k}}$. It remains to show that $l_{h} F_{\nu_{h}} \geqslant F$. If $F(x, y)=-\infty$ there is nothing to prove, so suppose that $F(x, y)>-\infty$. We have to show that for the sequence $\left\{F_{\nu_{k}}\right.$, $k=1, \ldots\}$ condition (3.7) is satisfied, i.e. given any subsequence of functions $\left\{F_{\nu_{p}}\right.$, $p=1, \ldots\} \subset\left\{F_{\nu_{k}}, k=1, \ldots\right\}$, any sequence $\left\{x_{p}, p=1, \ldots\right\}$ converging to $x$, there exists a sequence $\left\{y_{p}, p=1, \ldots\right\}$ converging to $y$ such that

$$
F(x, y) \leqslant \liminf _{p \rightarrow \infty} F_{v_{p}}\left(x_{p}, y_{p}\right) \text {. }
$$

Since for each $q, F^{q}=\operatorname{lm}_{e / h} F_{p}^{q} \leqslant l_{h} F_{\nu_{p}}^{q}$, given $\left\{x_{p}, p=1, \ldots\right\}$ converging to $x$, there exists a subsequence $\left\{y_{p}^{q}, p=1, \ldots\right\}$ converging to $y$ such that

$$
F^{q}(x, y) \leqslant \liminf _{p \rightarrow \infty} F_{\nu_{p}}^{q}\left(x_{p}, y_{p}^{q}\right)
$$


The proof will be complete if we can find a subsequence $\left\{y_{p}=q_{p}^{q(p)}, p=1, \ldots\right\}$ that satisfies (6.3).

Now recall that $F(x, y)>-\infty$ and that

$$
F(x, y) \leqslant F^{q}(x, y) \leqslant \liminf _{p \rightarrow \infty} F_{\nu_{p}}^{q}\left(x_{p}, y_{p}^{q}\right)=\liminf _{p \rightarrow \infty} \sup \left[F_{\nu}\left(x_{p}, y_{p}^{q}\right),-q\right] .
$$

Thus for any $-q<F(x, y)$, we have that

$$
F(x, y) \leqslant F^{q}(x, y) \leqslant \liminf _{p \rightarrow \infty} F_{\nu_{p}}\left(x_{p}, y_{p}^{q}\right)
$$

and thus by definition of $F$,

$$
F(x, y) \leqslant \liminf _{q \rightarrow \infty} \liminf _{p \rightarrow \infty} F_{\nu_{p}}\left(x_{p}, y_{p}^{q}\right) .
$$

The same diagonalization procedure that allowed us to complete the proof of Step 2, yields the required subsequence.

Appendix. We prove here a number of nonstandard diagonalization lemmas.

A.1 Lemma. Let $\left\{a_{\nu, \mu}, \nu=1, \ldots, \mu=1, \ldots\right\}$ be a doubly indexed family in $\bar{R}$. Then there exists a map $\nu \rightarrow \mu(\nu)$ such that

$$
\liminf _{\nu \rightarrow \infty} a_{\nu, \mu(\nu)} \geqslant \liminf _{\mu \rightarrow \infty}\left(\liminf _{\nu \rightarrow \infty} a_{\nu, \mu}\right) .
$$

Proof. Let $a_{\mu}=\liminf _{\nu \rightarrow \infty} a_{\nu, \mu}$ and $a=\liminf _{\mu \rightarrow \infty} a_{\mu}$. First assume that $a<+\infty$. By definition of $a$, there exists $\mu_{1}$ such that

$$
a_{\mu} \geqslant a-1 / 2 \text { for all } \mu \geqslant \mu_{1}, \quad a_{\mu_{1}} \in R
$$

and by definition of $a_{\mu_{1}}$, there exists $\nu_{1}$ such that

$$
a_{\nu, \mu_{1}} \geqslant a_{\mu_{1}}-1 / 2 \text { for all } \nu \geqslant \nu_{1} .
$$

We now build two sequences $\left\{\mu_{p}, p=1, \ldots\right\}$ and $\left\{\nu_{p}, p=1, \ldots\right\}$ in the following manner:

$$
\mu_{p}>\mu_{p-1} \text { and } a_{\mu} \geqslant a-2^{-p} \text { for all } \mu \geqslant \mu_{p}
$$

and

$$
\nu_{p} \geqslant \nu_{p}, \quad \text { and } \quad a_{\nu, \mu_{p}} \geqslant a_{\mu_{p}}-2^{-p} \text { for all } \nu \geqslant \nu_{p} .
$$

Set $\mu(\nu)=\mu_{p}$ if $\nu_{p} \leqslant \nu \leqslant \nu_{p+1}$. One can verify that whenever $\nu_{p} \leqslant \nu \leqslant \nu_{p+1}$, we have

$$
a_{v, \mu\left(v^{\prime}\right)}-a=a_{\mu_{p}}^{v}-a_{\mu_{p}}+a_{\mu_{p}}-a \geqslant-2^{1-p} .
$$

Hence for all $\nu \geqslant \nu_{p}, a_{\nu, \mu(\nu)} \geqslant a-2^{1-p}$, from which it follows that $\liminf _{\nu \rightarrow \infty} a_{\nu, \mu(\nu)}$ $\geqslant a-2^{1-p}$. The fact that this holds for every $p$ yields (A.2).

Now let us consider the situation when $a=+\infty$. This implies that to any number $p$ there corresponds $\mu_{p}$ such that for all $\mu \geqslant \mu_{p}, a_{\mu} \geqslant p$. By definition of $a_{\mu_{p}}$ this means that $a_{\nu, \nu_{p}} \geqslant p-p^{-1}$ for all $\nu \geqslant \nu_{p}$. The sequence $\left\{a_{l, \mu(l)}, l=1, \ldots\right\}$ is built by the following algorithm. 


$$
\begin{array}{ll}
a_{1, \mu(1)}=a_{1,1}, & \text { set } p=1, \quad l=1, \\
\text { if } \nu_{p}>l+1, & \text { set } a_{l+1, \mu(l+1)}=a_{l+1, \mu(l)}, \\
& \text { set } l=l+1 \text { and return to }(*), \\
\text { if } \nu_{p} \leqslant l+1, & \text { set } a_{l+1, \mu(l+1)}=a_{l+1, \mu_{p}}, \\
& \text { set } p=p+1, l=l+1 \text { and return to }(*)
\end{array}
$$

where $\nu_{p}$ and $\mu_{p}$ are as defined above. It is easy to see that $\lim _{l \rightarrow \infty} a_{l, \mu(l)}=+\infty$.

A.3 Corollary. Let $\left\{a_{\nu, \mu}, \nu=1, \ldots, \mu=1, \ldots\right\}$ be a doubly indexed family in $\bar{R}$. Then there exists a map $\nu \mapsto \mu(\nu)$ such that

$$
\limsup _{\nu \rightarrow \infty} a_{\nu, \mu(\nu)} \leqslant \limsup _{\mu \rightarrow \infty}\left(\limsup _{\nu \rightarrow \infty} a_{\nu, \mu}\right) .
$$

A.5 Lemma. Let $\{a(\nu, \cdot) ; \nu=1, \ldots\}$ be a sequence of extended-real valued functions defined on $X$, a general space. Then, there exists a map $\nu \mapsto x(\nu)$ such that

$$
\liminf _{\nu \rightarrow+\infty} \sup _{x \in X} a(\nu, x) \leqslant \liminf _{\nu \rightarrow+\infty} a(\nu, x(\nu))
$$

and

$$
\limsup _{\nu \rightarrow+\infty} \sup _{x \in X} a(\nu, x) \leqslant \limsup _{\nu \rightarrow+\infty} a(\nu, x(\nu)) .
$$

Proof. Let us define for each $\nu \in N, x(\nu) \in X$ in the following way.

If $\sup _{x \in \mathrm{X}} a(\nu, x)<+\infty$, take $x(\nu) \in X$ such that

$$
\sup _{x \in X} a(\nu, x) \leqslant a(\nu, x(\nu))+v^{-1} .
$$

If $\sup a(\nu, x)=+\infty$, take $x(\nu) \in X$ such that

$$
\nu \leqslant a(\nu, x(\nu)) \text {. }
$$

We claim that with this choice of the $x(\nu),($ A.6) holds: if the right member of (A.6) is equal to $+\infty$, there is nothing to prove. So let us assume $\liminf _{\nu \rightarrow+\infty} a(\nu, x(\nu))<$ $+\infty$. By definition of the lower limit there exists a subsequence $\left\{\nu_{k} ; k=1, \ldots\right\}$ such that

$$
\liminf _{\nu \rightarrow+\infty} a(\nu, x(\nu))=\lim _{k \rightarrow+\infty} a\left(\nu_{k}, x\left(\nu_{k}\right)\right)<+\infty .
$$

From the definition of $x(\nu)$, this implies that for $k$ sufficiently large

$$
\sup _{x \in X} a\left(\nu_{k}, x\right)<+\infty \quad \text { and } \sup _{x \in X} a\left(\nu_{k}, x\right) \leqslant a\left(\nu_{k}, x\left(\nu_{k}\right)\right)+\nu_{k}^{-1} .
$$

Finally,

$$
\begin{aligned}
\liminf _{\nu \rightarrow+\infty} a(\nu, x(\nu)) & =\lim _{k \rightarrow+\infty} a\left(\nu_{k}, x\left(\nu_{k}\right)\right) \geqslant \lim _{k} \inf \sup _{x \in X} a\left(\nu_{k}, x\right) \\
& \geqslant \liminf _{\nu \rightarrow+\infty} \sup _{x \in X} a(\nu, x) .
\end{aligned}
$$

Now let us prove (A.7). As for (A.6) the only case we have to consider is when $\lim \sup _{\nu \rightarrow+\infty} a(\nu, x(\nu))<+\infty$. This implies that there exists a positive real number $r_{0}$ and an index $\nu_{0}$ such that

$$
\text { for all } \nu \geqslant \nu_{0} \quad a(\nu, x(\nu)) \leqslant r_{0} .
$$


From the definition of $x(\nu)$, this implies that for $\nu>\sup \left\{\nu_{0}, r_{0}\right\}$

$$
\sup _{x \in X} a(\nu, x)<+\infty \quad \text { and } \sup _{x \in X} a(\nu, x) \leqslant a(\nu, x(\nu))+\nu^{-1} .
$$

Taking the upper limit with respect to $\nu$, we obtain

$$
\limsup _{\nu \rightarrow+\infty} \sup _{x \in X} a(\nu, x) \leqslant \limsup _{\nu \rightarrow+\infty} a(\nu, x(\nu)) .
$$

A.8 Corollary. Consider the sequence $\{a(\nu, \cdot): X \rightarrow \bar{R} ; \nu=1, \ldots\}$. Then, there exists a map $\nu \mapsto x(\nu)$ such that

$$
\liminf _{\nu \rightarrow+\infty} \inf _{x \in X} a(\nu, x) \geqslant \liminf _{\nu \rightarrow+\infty} a(\nu, x(\nu)) .
$$

Acknowledgement. We thank G. Buttazzo and G. Greco for pointing out that only sup, and not max, is valid in expression (4.36).

\section{REFERENCES}

1. H. Attouch, Families d'opérateurs maximaux monotones et mesurabilité, Ann. Mat. Pura Appl. 120 (1979), 35-111.

2. H. Attouch and R. Wets, Approximation and convergence in nonlinear optimization, Nonlinear Programming, vol. 4 (O. Mangasarian, R. Meyer and S. Robinson, eds.), Academic Press, New York, 1981.

3. , Convergence of convex-concave saddle functions (in preparation).

4. E. Cavazzuti, $\Gamma$-convergenze multiple, convergenza di punti di sella e di max-min, Boll. Un. Mat. Ital. (to appear).

5. E. De Giorgi, Convergence problems for functionals and operators, Recent Methods in Nonlinear Analysis, Pitagora Editrice, Bologna, 1979, pp. 131-138.

6. E. De Giorgi, G. Dal Masso and P. Longo, $\Gamma$-limiti di ostacoli, Atti Acad. Naz. Lincei Rend. Cl. Sci. Fis. Mat. Natur. (to appear).

7. S. Dolecki, G. Salinetti and R. Wets, Convergence of functions: Equi-semicontinuity, Trans. Amer. Math. Soc. 276 (1983), 409-429.

8. C. Kuratowski, Topologie. I, PWN, Warsaw, 1958.

9. U. Mosco, Convergence of convex sets and solutions of variational inequalities, Adv. in Math. 3 (1969), 510- 585 .

10. R. T. Rockafellar, Minimax theorems and conjugate saddle functions, Math. Scand. 14 (1964), 151-173.

11. G. Salinetti and R. Wets, On the convergence of closed sets, Topology Proc. 4 (1979), 149-158.

12. Y. Sonntag, Convergence au sens de U. Mosco. Meilleure approximation, applications de dualité, Exposé multigraphié, Sém Math. Appl., Univ. de Provence, 1980.

13. R. Wets, Convergence of convex functions, variational inequalities and convex optimization problems, Variational Inequalities and Complementarity Problems (R. Cottle, F. Gianessi and J.-L. Lions, eds.), Wiley, London, 1980, pp. 375-403.

14. T. Zolezzi, Variational stability and well-posedness in the optimal control of ordinary differential equations, Technical Report, Universita di Genova, 1981

\section{Mathematiques, Orsay, Paris, France}

DePaRTMent of MATHematics, University of Kentucky, LeXington, KentuCKy 40506 